\title{
Unraveling a new lineage of Hydrobiidae genera (Caenogastropoda: Truncatelloidea) from the Ponto-Caspian region
}

\author{
Diana DELICADO ${ }^{1, *}$, Vladimir PEŠIĆ ${ }^{2} \&$ Peter GLÖER $^{3}$ \\ ${ }^{1}$ Justus Liebig University Giessen, Department of Animal Ecology \& Systematics, \\ Heinrich-Buff-Ring 26-32 IFZD-35392, Giessen, Germany. \\ ${ }^{2}$ University of Montenegro, Department of Biology, 81000, Podgorica, Montenegro. \\ ${ }^{3}$ Biodiversity Research Laboratory, Schulstr. 3, D-25491, Hetlingen, Germany. \\ ${ }^{*}$ Corresponding author: didelicado@gmail.com \\ ${ }^{2}$ Email: vladopesic@gmail.com \\ ${ }^{3}$ Email: gloeer@malaco.de \\ ${ }^{1}$ urn:1sid:zoobank.org:author:52A050CB-4AE4-4EEF-8D5E-B508A0EC5AB2 \\ ${ }^{2}$ urn:lsid:zoobank.org:author:B4FDC912-E185-43E0-868D-95BA1918576E \\ ${ }^{3}$ urn:1sid:zoobank.org:author:8CB6BA7C-D04E-4586-BA1D-72FAFF54C4C9
}

\begin{abstract}
Phylogenetic analyses of the highly diverse (non-marine aquatic) gastropod family Hydrobiidae Stimpson, 1865 have revealed seven main lineages, most of which represent subfamilies. The subfamily Pseudamnicolinae Radoman, 1977, and specifically the genus Pseudamnicola Paulucci, 1878 (mainly inhabiting western and central Mediterranean regions), contributes substantially to this hydrobiid richness. Most of its congeners have been described in terms of their shell and penis features, which are of limited diagnostic value. Hence, the taxonomic status of some Pseudamnicola species needs to be revised, particularly of those inhabiting marginal regions, such as the Ponto-Caspian domain, largely occupied by the subfamily Pyrgulinae Brusina, 1882. Here we present a molecular phylogeny including species of both subfamilies along with extended morphological descriptions to confirm assignments of the Iranian species Pseudamnicola zagrosensis Glöer \& Pešić, 2009; Sarkia kermanshahensis Glöer \& Pešić, 2009 (originally within Pseudamnicola) and P. saboori Glöer \& Pešić, 2009. Our COI-based tree rejects these assignments suggesting a new potential lineage, sister to the pyrgulinid species, and comprising three genera: Shadinia Akramowski, 1976, Intermaria gen. nov. and Persipyrgula gen. nov. These genera differ molecularly by $3.6 \%-8.5 \%$, and are diagnosable by penis, female genitalia and radula features. Our findings evidence the high morphological variability of pyrgulinid species and provide insight into the origins and evolution of the freshwater Ponto-Caspian fauna.
\end{abstract}

Keywords. Freshwater snails, Pseudamnicola, Cryptic taxa, Anatomy, mtDNA.

Delicado D., Pešić V. \& Glöer P. 2016. Unraveling a new lineage of Hydrobiidae genera (Caenogastropoda: Truncatelloidea) from the Ponto-Caspian region. European Journal of Taxonomy 208: 1-29. http://dx.doi. org/10.5852/ejt.2016.208 


\section{Introduction}

The family Hydrobiidae Stimpson, 1865 (sensu Wilke et al. 2013) is thought to be the most diverse family of non-marine aquatic gastropods, with ca 70 genus-level and 550 species-level taxa. Phylogenetic analyses have recovered seven main lineages within this family, most of which represent subfamilies (see Wilke et al. 2013). Among the genera contributing most to this remarkable richness is the genus Pseudamnicola Paulucci, 1878 (subfamily Pseudamnicolinae Radoman, 1977). Despite the promotion of its former congeneric subgenus Corrosella Boeters, 1970 to the genus level (see Delicado et al. 2015), Pseudamnicola still comprises around 70 nominal species distributed mainly across the western and central Mediterranean region (Glöer et al. 2015). However, this richness may be overestimated due to erroneous assignments to the genus since most of its species have been diagnosed according only to a few shell features or at most to both shell and penis descriptions. Pseudamnicola species, like most hydrobiids, are characterized by a minute, unsculpted shell and simple penis, such that only comprehensive studies including the description of several anatomical structures and molecular data have been able to distinguish among congeners (Hershler \& Ponder 1998; Wilke et al. 2001; Arconada \& Ramos 2003; Strong et al. 2008). Morphological and molecular data may offer varying resolution in cases of cryptic species (Wilke et al. 2002; Liu et al. 2003; Delicado \& Ramos 2012) or when high morphological disparity exists among close related taxa (Wilke et al. 2007; Delicado et al. 2014). Thus, unraveling the systematics of the hydrobiids family calls for an integrative approach.

Although a systematic revision of Pseudamnicola is still pending, Delicado et al. (2015) revealed the monophyly of Pseudamnicola s. str. and proposed a series of diagnostic characters including a simple ovate-conic shell shape, single elongated seminal receptacle and broadly triangular penis with several folds across its surface. Hence, we propose the revision of the taxonomic status of some Pseudamnicola species originally described according to shell features only. These include species inhabiting PontoCaspian regions, marginal areas of the family's main distribution range, such as Iran (Glöer \& Pešić 2009, 2012), Turkey (Yıldırım et al. 2006; Glöer \& Georgiev 2012; Glöer et al. 2014, 2015) and Georgia (Badzoshvili 1979). One such species Sarkhia kermanshahensis (Glöer \& Pešić, 2009) has been recently assigned by Glöer \& Pešić (2012) to an independent genus of unclear phylogenetic relationship. Freshwater systems in these areas are mainly occupied by members of the subfamily Pyrgulinae (see Radoman 1983; Wilke et al. 2007). These share some morphological and anatomical similarities with Pseudamnicola (e.g., complete ctenidium, yellowish, oval operculum, pigmented renal oviduct and nervous system), yet they also differ in terms of other features (e.g., shell shape and sculpture, penis shape, number of seminal receptacles, number of basal cusps in the radular central tooth, etc.) and also appear relatively distant from Pseudamnicola in the Hydrobiidae phylogeny (Wilke et al. 2013).

Through a molecular/anatomical approach, the present study revises the taxonomic status of the Pseudamnicola species from Iran described by Glöer \& Pešić (2009), i.e., P. zagrosensis Glöer \& Pešić, 2009, Sarkhia kermanshahensis (Glöer \& Pešić, 2009) (originally described as Pseudamnicola kermanshahensis) and P. saboori Glöer \& Pešić, 2009. We also examined whether the springsnail genus Shadinia Akramowski, 1976, cited only from a few localities of Iran's neighbour Armenia, anatomically and molecularly resembles the pyrgulinid taxa. So far, three species of this genus have been described based both on shell characteristics (Shadin 1952; Egorov 2006) and some anatomical structures (Akramovski 1976: figs 29, 30; Glöer et al. 2016). Their phylogenetic relationships within the family Hydrobiidae, nevertheless, remain unclear. Accordingly, we here examine several anatomical structures of the type species of Shadinia, S. terpoghassiani (Shadin, 1952), and obtained partial gene sequences in this and the recently described species S. bjniensis Glöer et al., 2016.

Our results indicate that the Pseudamnicola species described by Glöer \& Pešić (2009) and the genus Shadinia are likely pyrgulinid taxa. This information provides clues about the actual biodiversity and biogeographic patterns of the hydrobiid subfamilies Pseudamnicolinae and Pyrgulinae, with possible 
DELICADO D. et al., New hydrobiid genera from the Ponto-Caspian region

implications for on-going and future research efforts targeted at understanding the origins and evolution of freshwater biodiversity, such as the projects PRIDE (Ponto-Caspian biodiversity Rise and Demise) or SCOPSCO (Scientific Collaboration On Past Speciation Conditions in Ohrid).

\section{Material and methods}

To assess the taxonomic status of the three Pseudamnicola species from Iran described in Glöer \& Pešić (2009), we examined molecular and anatomical data of the original material collected by these authors, together with a sample of Shadinia terpoghassiani (type species) collected from the type locality (Lake

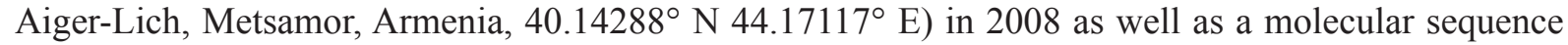
of Shadinia bjniensis. The Shadinia terpoghassiani sample is preserved in $80 \%$ ethanol and deposited in the biodiversity collection at Justus Liebig University Giessen (UGSB). The third described species, Shadinia akramowskii (Shadin, 1952), could not be relocated yet.

Total DNA obtained here de novo was isolated from one individual per species following the CTAB protocol of Wilke et al. (2006). A partial 658-bp of the mitochondrial fragment cytochrome $c$ oxidase subunit I (COI) was PCR amplified with the primers LCO1490 and HCO2198 (Folmer et al. 1994). Thermal cycling conditions were as described in Delicado et al. (2012) and were conducted with an annealing temperature of $48^{\circ} \mathrm{C}$. Final PCR products were sequenced in an ABI $3730 \mathrm{XL}$ sequencer (Life Technologies, Carlsbad, CA, USA) using a Big Dye Terminator kit v. 3.1 (Life Technologies). The new sequences were deposited in GenBank under the accession numbers indicated in Table 1.

DNA sequences were edited in Sequencher 4.6 (Gene Codes, Ann Arbor, MI) and aligned manually in PAUP* 4.0a123 (Swofford 2002) together with the sequences of other related hydrobiid species obtained from GenBank (Table 1). Phylogenetic reconstruction was conducted using maximum likelihood (ML) and Bayesian-inference (BI). ML analysis was performed in PHYML v3.0 (Guindon \& Gascuel 2003) using the evolutionary model selected in jModelTest v. 2.1.4 (Darriba et al. 2012) under corrected Akaike's information criterion (Akaike 1974; Sugiura 1978; Hurvich \& Tsai 1989). The BI was run through two independent runs of four Metropolis-coupled chains in MrBayes 3.1.2 (Huelsenbeck 2000; Huelsenbeck \& Ronquist 2001), with 5 million generations each and a sample frequency of 1000. The analysis was terminated when the standard deviation of split frequencies reached values of $<0.01$ in MrBayes 3.1.2. Convergence between runs was in addition monitored by reviewing that each posterior parameter reached values of effective sample size, estimated in Tracer 1.5 (Rambaut \& Drummond 2009), greater than 200. The first 10\% sampled trees were discarded as burn-in. The robustness of the inferred topologies was assessed by bootstrapping (Felsenstein 1985) with 1000 pseudoreplicates in $\mathrm{ML}$, and by posterior probabilities (BPPs) of Bayesian trees.

The number of the dissected specimens and their respective localities are indicated in Tables 2 and 3. Dissections and measurements were made with a Keyence VHX-2000E digital microscope in combination with the program VHX-2000 Communication software version 2.3.5.0 (Keyence Corporation, 20092012). Radulae were extracted from buccal mass by applying the first step of the established Proteinase $\mathrm{K}$ protocol for DNA isolation (Wilke et al. 2006). After mounting on stubs and drying, radulae were sputter coated with gold (Balter Sputter Coater SCD004) for $50 \mathrm{sec}$. in order to photograph them with a field emission scanning electron microscope (FESEM) DSM982 Gemini (Carl Zeiss GmbH, Germany). Morphological character states are based on the terminology of Hershler \& Ponder (1998). Whorls were counted according to the method of Ramos et al. (2000). The concentration of the nervous system was calculated as the RPG ratio (Davis et al. 1976) and also characterized using the categories of Davis et al. $(1984,1986,1992)$ as follows: dorsal nerve ring concentrated $(\leq 0.29)$; moderately concentrated $(0.30-0.49)$; elongated (0.50-0.67); extremely elongated $(\geq 0.68)$. Analysis of variance (ANOVA) was applied to test for statistical significance among morphological dimensions of the Ponto-Caspian species studied here. These calculations have been done using the package MBESS (Kelley \& Lai 2011) for the 
Table 1. Locality name, GenBank accession numbers and original references for the species employed in the molecular study.

\begin{tabular}{|c|c|c|c|}
\hline Taxon & Locality & $\begin{array}{l}\text { GenBank \# } \\
\text { COI }\end{array}$ & Original reference \\
\hline \multicolumn{4}{|l|}{ Outgroup } \\
\hline Mercuria similis & $\begin{array}{l}\text { Italy, Friuli-Venetia-Julia, Udine, } \\
\text { Aquileia, Canale Panigai }\end{array}$ & AF367646 & Wilke et al. 2001 \\
\hline \multicolumn{4}{|l|}{ Hydrobiinae } \\
\hline Hydrobia acuta & France, Hérault, Etang du Prévost & AF278808 & Wilke et al. 2000 \\
\hline Peringia ulvae & Russia, Lagoon 'Levin navolok' & AF118302 & Wilke \& Davis 2000 \\
\hline Salenthydrobia ferrerii & $\begin{array}{l}\text { Italy, Lecce, Porto Cesareo, Torre } \\
\text { Lapillo, Bambinello Spring }\end{array}$ & AF449205 & Wilke 2003 \\
\hline Ecrobia ventrosa & Great Britain, Snettisham Lagoon & AF118335 & Wilke \& Davis 2000 \\
\hline \multicolumn{4}{|l|}{ Pseudamnicolinae } \\
\hline Pseudamnicola lucensis & $\begin{array}{l}\text { Italy, Tuscany, Bagni di Lucca, } \\
\text { Bagni Caldi, thermal spring }\end{array}$ & AF367651 & Wilke et al. 2001 \\
\hline Corrosella falkneri & $\begin{array}{l}\text { Spain, Granada, Orce, La Armada } \\
\text { spring }\end{array}$ & JF312224 & Delicado et al. 2012 \\
\hline Diegus gasulli & $\begin{array}{l}\text { Spain, Almería, Rambla de } \\
\text { Retamar }\end{array}$ & KF060743 & Delicado et al. 2014 \\
\hline \multicolumn{4}{|l|}{ Pyrgulinae } \\
\hline Chilopyrgula sturanyi & $\begin{array}{l}\text { Macedonia, Lake Ohrid, S of } \\
\text { Sveti Zaum }\end{array}$ & EF379284 & Wilke et al. 2007 \\
\hline Dianella thiesseana & $\begin{array}{l}\text { Greece, Lake Trichonis at Loutres } \\
\text { Mirtias }\end{array}$ & AY676127 & Wilke et al. 2007 \\
\hline Euxinipyrgula milachevitchi & $\begin{array}{l}\text { Russia, Sea of Azov, Miusski } \\
\text { Liman }\end{array}$ & EF379290 & Wilke et al. 2007 \\
\hline Falsipyrgula pfeiferi & Turkey, Isparta, Lake Eĝirdir & EF379296 & Wilke et al. 2007 \\
\hline Ginaia munda munda & Macedonia, Lake Ohrid & JN398637 & $\begin{array}{l}\text { Schreiber et al. } \\
2012\end{array}$ \\
\hline Ginaia munda sublitoralis & Macedonia, Lake Ohrid & JN398630 & $\begin{array}{l}\text { Schreiber et al. } \\
2012\end{array}$ \\
\hline Intermaria kermanshahensis & $\begin{array}{l}\text { Iran, Kermanshah Province, spring } \\
\text { between Sarab and Sahneh city }\end{array}$ & KT896670 & Present study \\
\hline Intermaria zagrosensis & $\begin{array}{l}\text { Iran, Kermanshah Province, Sar } \\
\text { Pol Kangarar village, Sar Pol } \\
\text { Kangarar stream }\end{array}$ & KT896669 & Present study \\
\hline
\end{tabular}


DELICADO D. et al., New hydrobiid genera from the Ponto-Caspian region

\begin{tabular}{|c|c|c|c|}
\hline Macedopyrgula pavlovici & Macedonia, Lake Ohrid & JN398635 & $\begin{array}{l}\text { Schreiber et al. } \\
\quad 2012\end{array}$ \\
\hline Macedopyrgula wagneri & Macedonia, Lake Ohrid & JN398617 & $\begin{array}{l}\text { Schreiber et al. } \\
\quad 2012\end{array}$ \\
\hline "Micromelania" lincta & Romania, Lake Razim, Sarichioi & EF379292 & Wilke et al. 2007 \\
\hline Ohridopyrgula macedonica & Macedonia, Lake Ohrid, Otesevo & EF379286 & Wilke et al. 2007 \\
\hline Persipyrgula saboori & $\begin{array}{l}\text { Iran, Khorrasan Province, Zou } \\
\text { Eram village, Zou Eram spring }\end{array}$ & KT896671 & Present study \\
\hline Pyrgula annulata & $\begin{array}{l}\text { Italy, Brescia, Lake Garda, } \\
\text { Desenzano del Garda }\end{array}$ & AY341258 & Wilke et al. 2007 \\
\hline Shadinia terpoghassiani & $\begin{array}{l}\text { Armenia, south of Metzamor, } \\
\text { lake Aiger-Lich outflow }\end{array}$ & KT896672 & Present study \\
\hline Shadinia bjniensis & $\begin{array}{l}\text { Armenia, Kotyak province, Bjni, } \\
\text { Hrazdan river }\end{array}$ & KT896673 & Present study \\
\hline Turricaspia sp. & $\begin{array}{l}\text { Ukraine, Kherson, lower Dnieper, } \\
\text { near hydrobiological station }\end{array}$ & EF379294 & Wilke et al. 2007 \\
\hline Xestopyrgula dybowskii & $\begin{array}{l}\text { Macedonia, Lake Ohrid, S of } \\
\text { Sveti, Zaum }\end{array}$ & EF379288 & Wilke et al. 2007 \\
\hline
\end{tabular}

R statistical environment (R Development Core Team 2011). Resulting parameter values are shown in Tables 2 and 3.

The localities are listed according to the code: spring or lake, city, province, country, co-ordinates, altitude (when measured) and date of collection.

\section{Abbreviations}

\section{Shell characters}

$\mathrm{AH}=$ aperture height

$\mathrm{AL}=$ aperture length

$\mathrm{AW}=$ aperture width

LBW = length of body whorl

$\mathrm{NSW}=$ number of shell whorls

$\mathrm{SL}=$ shell length

SW $=$ shell width

WAW $=$ width of the antepenultimate whorl

WBW $=$ width of the body whorl

WPW $=$ width of the penultimate whorl

\section{Anatomical characters}

$\mathrm{Ag}=$ albumen gland

$\mathrm{Bc}=$ bursa copulatrix

$\mathrm{Cg}=$ capsule gland

$\mathrm{Ct}=$ ctenidium

$\mathrm{dBc}=$ duct of the bursa copulatrix 


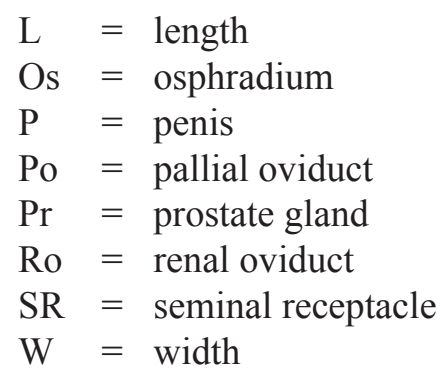

\section{Collections}

UGSB = Biodiversity collection at Justus Liebig University Giessen

$\mathrm{ZMH}=$ Zoologisches Museum Hamburg

\section{Results}

Phylogenetic inferences were based on $658 \mathrm{bp}$ of the COI gene under the nucleotide substitution model TrN (Tamura \& Nei 1993) + I (invariable sites) + G (rate variation among sites). Average base frequencies for the data set were $27.4 \% \mathrm{~A}, 14.1 \% \mathrm{C}, 15 \% \mathrm{G}$ and $43.5 \% \mathrm{~T}$. Both tree topologies (ML and BI) indicated that the Pseudamnicola species from the Ponto-Caspian region formed a monophyletic group independent of the subfamily Pseudamnicolinae (Fig. 1). However, this monophyly was well supported by ML (bootstrap value $=77 \%$ ) but less supported by BI $(\mathrm{BPP}=0.84)$. This newly discovered lineage appeared as sister to the pyrgulinid clade with high support ( $85 \%$ of bootstrap in ML and 1.00 of $\mathrm{PP}$ in BI). Species of Shadinia also grouped within this newly discovered lineage as sister to the species P. zagrosensis and Sarkhia kermanshahensis. The close relationship between the latter taxa suggests they could both belong to the same genus. Additionally, Shadinia and P. saboori may constitute two different genera. Sequence differences between species of this lineage (uncorrected pairwise distance, p-distance) ranged from $0.1 \%$ to $8.5 \%$ for COI (Table 4 ), and mean divergences between this lineage and the Pseudamnicolinae and Pyrgulinae species were $13.6 \%$ and $13 \%$, respectively.

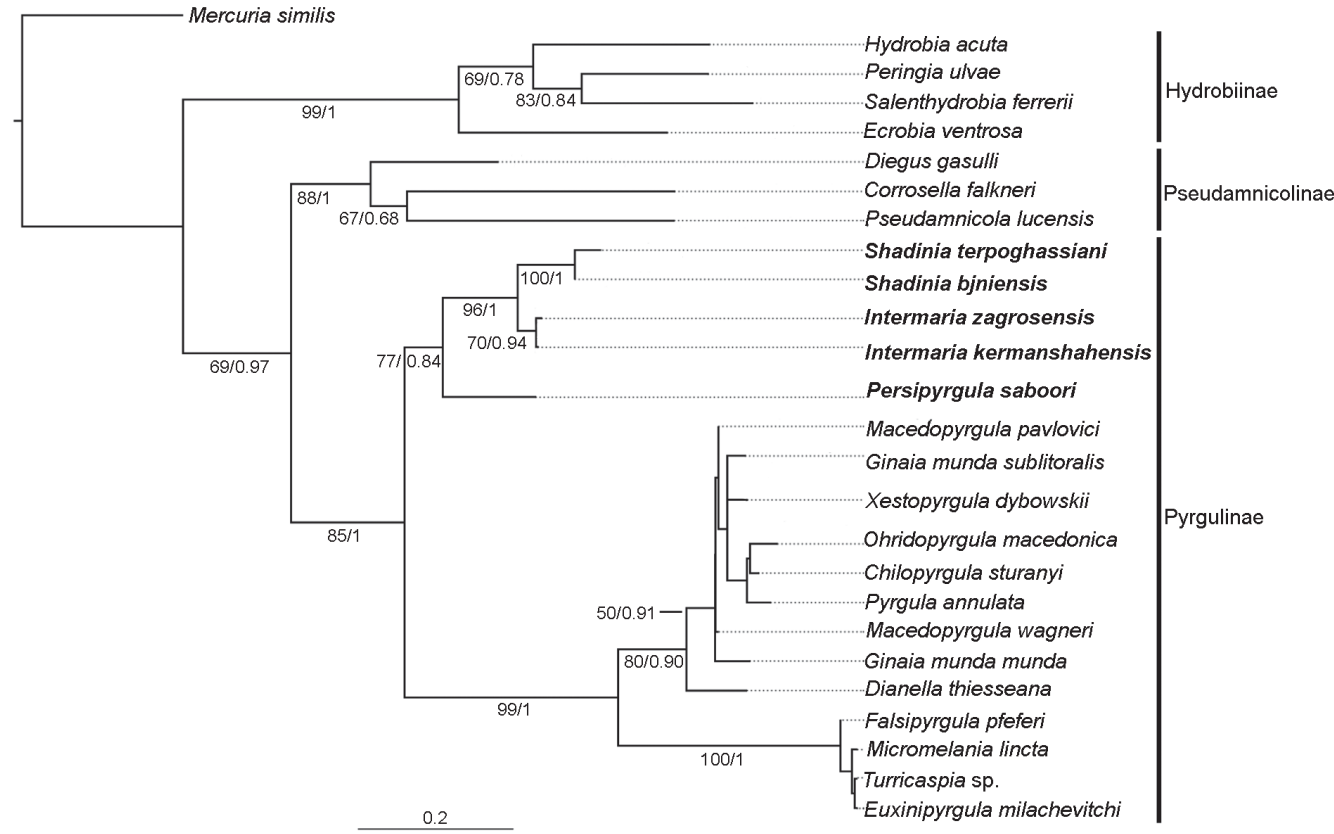

Fig. 1. Bayesian inference of hydrobiid species based on COI sequences. Values below branches indicate bootstrap supports for maximum likelihood and BPPs for Bayesian inference. Black bars on the right denote subfamily assignments. Scale bar: expected change per site. 
Morphological data also supported the existence of three different groups, which are hereafter treated as independent genera within the newly recovered hydrobiid lineage. The inclusion of this group within one or other subfamily is discussed in the following section.

Class Gastropoda (Cuvier, 1797)

Superorder Caenogastropoda Cox, 1960

Superfamily Truncatelloidea Gray, 1840

Family Hydrobiidae Stimpson, 1865

Genus Intermaria gen. nov.

urn:Isid:zoobank.org:act:1CDF6CD1-5D3C-447F-BAAE-7197FBEB2614

\section{Diagnosis}

Shell ovate-conic, 3 to $4.5 \mathrm{~mm}$ high; large and convex body whorl; rest of whorls small and slightly convex; aperture pyriform, angled on top and often fused to the body whorl. Operculum corneous, yellowish, thin, pliable, ellipsoidal, paucispiral with submarginal nucleus. Two pairs of basal cusps on radular central tooth. Ctenidium occupying nearly the entire length of pallial cavity bearing welldeveloped gill filaments. Osphradium opposite approximate middle of ctenidium. Bursa copulatrix lying against the middle section of the albumen gland; pigmented renal oviduct; one elongate seminal receptacle. Prostate gland bean-shaped, about twice as long as wide; exit of the pallial vas deferens from the posterior-most section of the prostate gland and seminal vesicle entering the prostate gland in its middle section; penis simple, gradually tapering, with its distal end tapered and often with a small distal lobe on the inner edge. Nervous system with black pigmentation typically elongated.

\section{Etymology}

From Latin inter- (between) -maria (seas), referring to the occurrence of the genus in the continental area between the Mediterranean and the Caspian seas.

\section{Type species}

Pseudamnicola zagrosensis Glöer \& Pešić, 2009.

\section{Remarks}

Intermaria species, like those of Pseudamnicola, have an ovate-conic simple shell, one elongated seminal receptacle and a pigmented renal oviduct and nervous system. However, Intermaria differs in having smaller shell dimensions (e.g., when compared with the $5 \mathrm{~mm}$ height of $P$. granjaensis Glöer \& Zettler, 2007 see Delicado et al. 2014), a more conic-shaped shell, two basal cusps on the central radular tooth (one in Pseudamnicola), a shorter prostate gland, and a gradually tapering penis with a small distal lobe on the inner edge (penis is triangular with many surface folds and has a blunt end in Pseudamnicola see Delicado et al. 2015).

Intermaria zagrosensis (Glöer \& Pešić, 2009) comb. nov.

Figs 2-3, Tables 2-3

Pseudamnicola zagrosensis Glöer \& Pešić, 2009: 37, pl. 6, figs 4-6.

\section{New diagnosis}

Shell ovate-conic, yellowish, with pyriform aperture; protoconch microsculpture wrinkled; central radular tooth formula (5)4-C-4(5)/2-2; pyriform bursa copulatrix; one seminal receptacle elongate with a short duct; penis gradually tapering, with a small distal lobe on inner edge, end tapered and grayish pigmented; nervous system elongated (mean RPG ratio $=0.60$ ) and slightly black pigmented. 


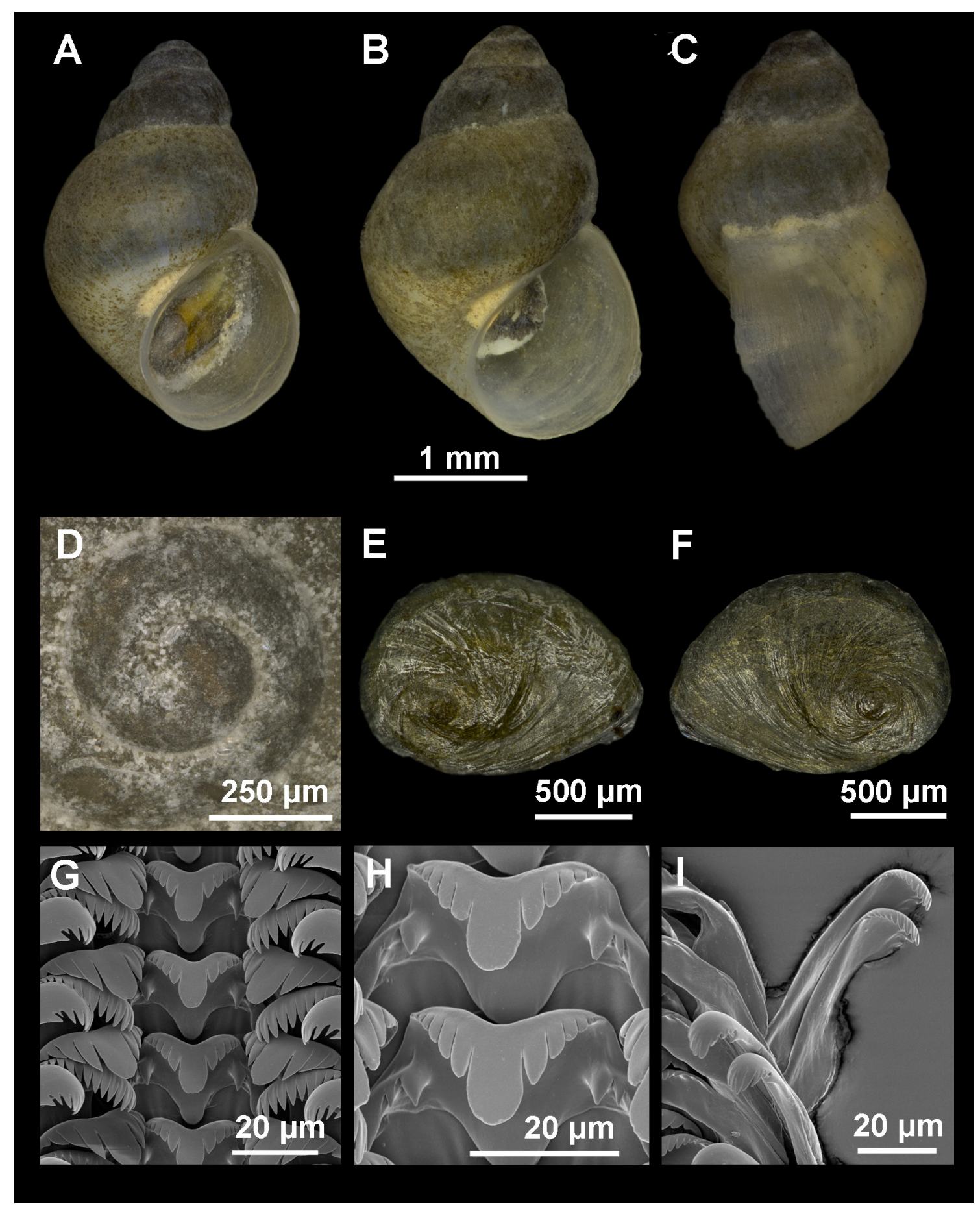

Fig. 2. Shell, operculum and radula of Intermaria zagrosensis (Glöer \& Pešić, 2009) gen. et comb. nov. from Sar Pol Kangarar stream, Kermanshah Province, Iran. A-B. Shell in front view. C. Shell in lateral view. D. Protoconch and microsculpture. E-F. Internal and external side of the operculum. G. Rows of teeth of the radula. H. Central teeth. I. Detail of outer marginal teeth. 


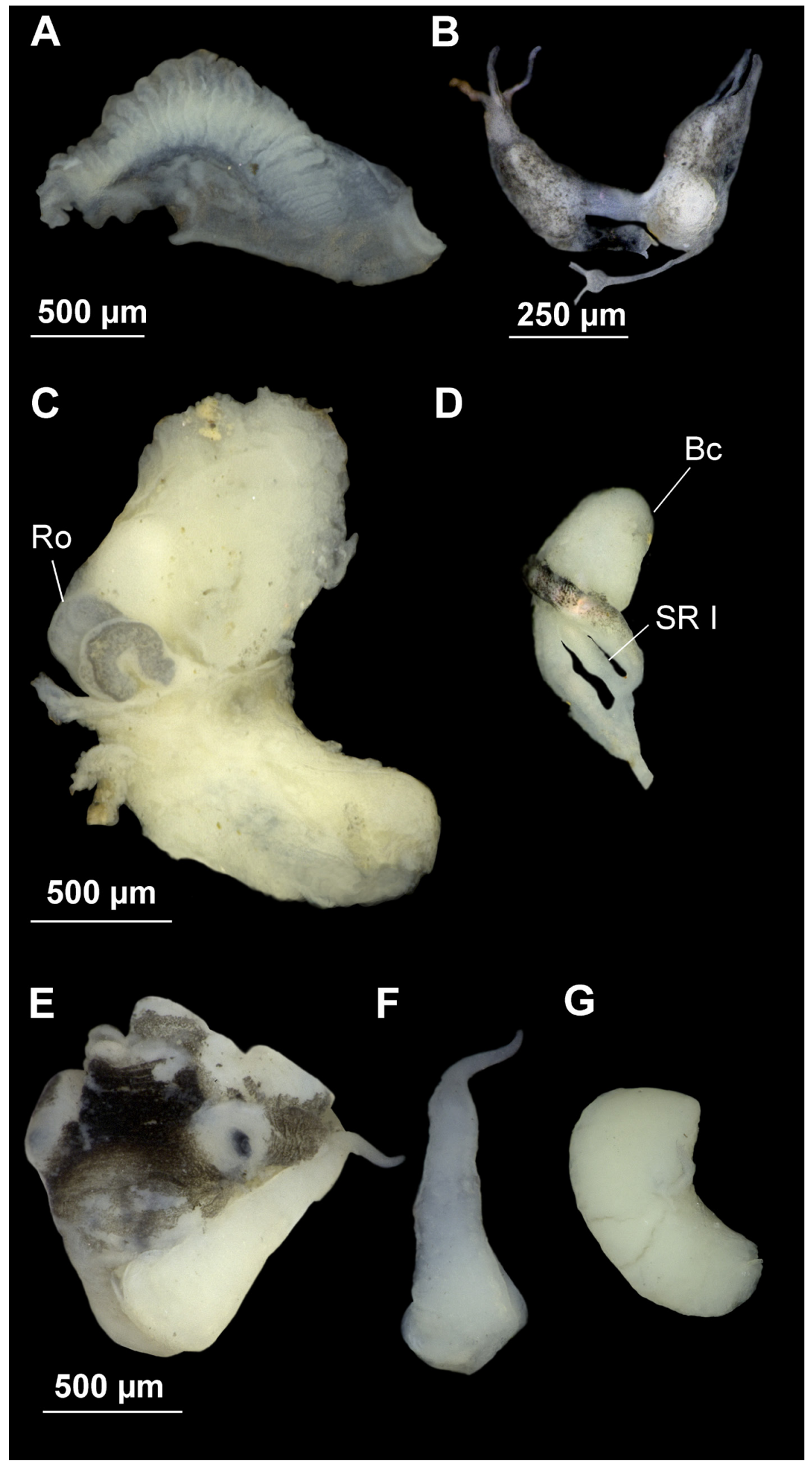

Fig. 3. Anatomy of Intermaria zagrosensis (Glöer \& Pešić, 2009) gen. et comb. nov. from Pol Kangarar stream, Kermanshah Province, Iran. A. Ctenidium and osphradium. B. Partial nervous system. C. Female genitalia. D. Bursa copulatrix and seminal receptacle. E-F. Head of a male and penis. G. Prostate gland. Anatomical abbreviations given in the Material and methods section. 
Table 2. Shell measurements (in mm) of the species: 1. Intermaria zagrosensis (Glöer \& Pešić, 2009). 2. Intermaria kermanshahensis (Glöer \& Pešić, 2009). 3. Persipyrgula saboori (Glöer \& Pešić, 2009). 4. Shadinia terpoghassiani (Shadin, 1952). ANOVA results are shown through effect size F, degrees of freedom (df), residuals (r) and the resulting significance (p-value: *** when 0 ; ** when 0.001 ; * when $0.01 ; \mathrm{NS}=$ no significant).

\begin{tabular}{|c|c|c|c|c|c|}
\hline & 1 & 2 & 3 & 4 & \\
\hline & $\begin{array}{c}\text { Mean } \pm \mathrm{SD} ; \mathrm{CV} \\
(\mathrm{Max}-\mathrm{Min}) \\
(\mathrm{n}=11)\end{array}$ & $\begin{array}{c}\text { Mean } \pm \mathrm{SD} ; \mathrm{CV} \\
(\mathrm{Max}-\mathrm{Min}) \\
(\mathrm{n}=7)\end{array}$ & $\begin{array}{c}\text { Mean } \pm \mathrm{SD} ; \mathrm{CV} \\
(\mathrm{Max}-\mathrm{Min}) \\
(\mathrm{n}=7)\end{array}$ & $\begin{array}{c}\text { Mean } \pm \text { SD; CV } \\
\quad(\text { Max-Min }) \\
(\mathrm{n}=20)\end{array}$ & $\begin{array}{c}\text { ANOVA } \\
\left(\mathrm{F}_{\mathrm{df}, \mathrm{r}}\right)\end{array}$ \\
\hline SL & $\begin{array}{c}3.58 \pm 0.40 ; 0.11 \\
(4.50-2.98)\end{array}$ & $\begin{array}{c}3.30 \pm 0.36 ; 0.11 \\
\quad(4.00-2.80)\end{array}$ & $\begin{array}{c}2.90 \pm 0.67 ; 0.23 \\
\quad(4.40-2.48)\end{array}$ & $\begin{array}{c}3.02 \pm 0.25 ; 0.08 \\
\quad(3.61-2.60)\end{array}$ & $\mathrm{F}_{3,40}=6.1^{* *}$ \\
\hline SW & $\begin{array}{l}2.28 \pm 0.33 ; 0.15 \\
\quad(3.00-1.74)\end{array}$ & $\begin{array}{c}2.00 \pm 0.13 ; 0.07 \\
\quad(2.70-1.77)\end{array}$ & $\begin{array}{c}1.84 \pm 0.52 ; 0.28 \\
\quad(3.00-1.48)\end{array}$ & $\begin{array}{c}1.68 \pm 0.14 ; 0.09 \\
(1.96-1.41)\end{array}$ & $\mathrm{F}_{3,40}=10.4 * * *$ \\
\hline SL/SW & $\begin{array}{c}1.58 \pm 0.10 ; 0.07 \\
\quad(1.71-1.45)\end{array}$ & $\begin{array}{c}1.58 \pm 0.05 ; 0.03 \\
\quad(1.66-1.50)\end{array}$ & $\begin{array}{c}1.59 \pm 0.08 ; 0.05 \\
(1.70-1.48)\end{array}$ & $\begin{array}{c}1.80 \pm 0.10 ; 0.06 \\
\quad(2.05-1.63)\end{array}$ & $\mathrm{F}_{3,40}=19.4 * * *$ \\
\hline $\mathrm{AH}$ & $\begin{array}{c}1.82 \pm 0.22 ; 0.12 \\
\quad(2.34-1.58)\end{array}$ & $\begin{array}{l}1.62 \pm 0.13 ; 0.08 \\
\quad(1.71-1.37)\end{array}$ & $\begin{array}{l}1.31 \pm 0.15 ; 0.11 \\
\quad(1.50-1.09)\end{array}$ & $\begin{array}{c}1.45 \pm 0.11 ; 0.08 \\
\quad(1.68-1.25)\end{array}$ & $\mathrm{F}_{3,37}=18.3 * * *$ \\
\hline SL-LBW & $\begin{array}{l}0.83 \pm 0.08 ; 0.10 \\
\quad(0.97-0.67)\end{array}$ & $\begin{array}{l}0.75 \pm 0.08 ; 0.10 \\
\quad(0.83-0.61)\end{array}$ & $\begin{array}{l}0.66 \pm 0.05 ; 0.07 \\
\quad(0.75-0.62)\end{array}$ & $\begin{array}{l}0.84 \pm 0.11 ; 0.14 \\
\quad(1.08-0.66)\end{array}$ & $\mathrm{F}_{3,37}=5.9 * *$ \\
\hline WBW & $\begin{array}{c}1.98 \pm 0.19 ; 0.09 \\
\quad(2.26-1.58)\end{array}$ & $\begin{array}{c}1.92 \pm 0.12 ; 0.06 \\
\quad(2.07-1.76)\end{array}$ & $\begin{array}{l}1.51 \pm 0.07 ; 0.04 \\
\quad(1.62-1.44)\end{array}$ & $\begin{array}{c}1.58 \pm 0.13 ; 0.08 \\
\quad(1.88-1.37)\end{array}$ & $\mathrm{F}_{3,37}=26.3 * * *$ \\
\hline $\mathrm{AL}$ & $\begin{array}{c}1.77 \pm 0.16 ; 0.09 \\
(2.11-1.53)\end{array}$ & $\begin{array}{c}1.67 \pm 0.11 ; 0.07 \\
\quad(1.79-1.54)\end{array}$ & $\begin{array}{c}1.27 \pm 0.12 ; 0.09 \\
(1.39-1.06)\end{array}$ & $\begin{array}{c}1.41 \pm 0.12 ; 0.08 \\
\quad(1.70-1.24)\end{array}$ & $\mathrm{F}_{3,37}=28.2 * * *$ \\
\hline AW & $\begin{array}{c}1.36 \pm 0.09 ; 0.07 \\
(1.50-1.18)\end{array}$ & $\begin{array}{c}1.23 \pm 0.10 ; 0.08 \\
\quad(1.35-1.06)\end{array}$ & $\begin{array}{c}0.97 \pm 0.05 ; 0.07 \\
\quad(1.06-0.90)\end{array}$ & $\begin{array}{c}1.03 \pm 0.08 ; 0.08 \\
\quad(1.13-0.86)\end{array}$ & $\mathrm{F}_{3,37}=41.9 * * *$ \\
\hline WPW & $\begin{array}{c}1.20 \pm 0.12 ; 0.10 \\
\quad(1.41-0.98)\end{array}$ & $\begin{array}{c}1.17 \pm 0.05 ; 0.04 \\
\quad(1.24-1.10)\end{array}$ & $\begin{array}{l}0.96 \pm 0.05 ; 0.05 \\
\quad(1.02-0.90)\end{array}$ & $\begin{array}{c}1.03 \pm 0.09 ; 0.08 \\
(1.24-0.9)\end{array}$ & $\mathrm{F}_{3,37}=13.9 * * *$ \\
\hline WAW & $\begin{array}{l}0.13 \pm 0.02 ; 0.18 \\
\quad(0.19-0.11)\end{array}$ & $\begin{array}{l}0.13 \pm 0.02 ; 0.19 \\
\quad(0.18-0.11)\end{array}$ & $\begin{array}{l}0.10 \pm 0.02 ; 0.20 \\
\quad(0.13-0.07)\end{array}$ & $\begin{array}{l}0.13 \pm 0.03 ; 0.24 \\
\quad(0.23-0.08)\end{array}$ & $\mathrm{F}_{3,37}=1.4^{\mathrm{NS}}$ \\
\hline NSW & $\begin{array}{c}4.15 \pm 0.21 ; 0.05 \\
\quad(4.50-4.00)\end{array}$ & $\begin{array}{c}4.37 \pm 0.14 ; 0.03 \\
\quad(4.50-4.25)\end{array}$ & $\begin{array}{c}4.20 \pm 0.25 ; 0.06 \\
\quad(4.50-4.00)\end{array}$ & $\begin{array}{c}4.13 \pm 0.17 ; 0.04 \\
\quad(4.50-4.00)\end{array}$ & $\mathrm{F}_{3,37}=2.6^{\mathrm{NS}}$ \\
\hline
\end{tabular}

\section{Material examined}

Holotype

IRAN: ZMH 51406: $4.5 \mathrm{~mm}$ height, $3.0 \mathrm{~mm}$ width.

\section{Paratypes}

IRAN: ZMH 51407 (5 ex.) and P. Glöer's (68 ex.) collection.

\section{Type locality}

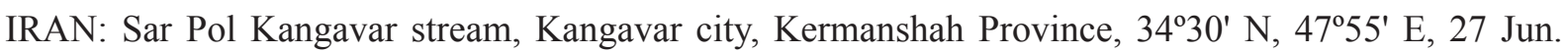
2005. 
DELICADO D. et al., New hydrobiid genera from the Ponto-Caspian region

Table 3. Ctenidium, osphradium. Female and male genitalia and nervous system measurements (in $\mathrm{mm}$ ) of the species: 1. Intermaria zagrosensis (Glöer \& Pešić, 2009). 2. Intermaria kermanshahensis (Glöer \& Pešić, 2009). 3. Persipyrgula saboori (Glöer \& Pešić, 2009). 4. Shadinia terpoghassiani (Shadin, 1952). For ANOVA results effect size F, degrees of freedom (df), residuals (r) and the resulting significance (p-value: $* * *$ when $0 ; * *$ when $0.001 ; *$ when $0.01 ; \mathrm{NS}=$ not significant) are shown.

\begin{tabular}{|c|c|c|c|c|c|}
\hline & $\begin{array}{c}1 \\
\text { Mean } \pm \text { SD; CV } \\
(\text { Max-Min) } \\
3+-2 \delta\end{array}$ & $\begin{array}{c}2 \\
\text { Mean } \pm \text { SD; CV } \\
(\text { Max-Min) } \\
1+-3 \sigma^{\lambda}\end{array}$ & $\begin{array}{c}\mathbf{3} \\
\text { Mean } \pm \mathrm{SD} ; \mathrm{CV} \\
(\mathrm{Max}-\mathrm{Min}) \\
2+-2 \hat{0}\end{array}$ & $\begin{array}{c}4 \\
\text { Mean } \pm \mathrm{SD} ; \mathrm{CV} \\
(\mathrm{Max}-\mathrm{Min}) \\
5+-30\end{array}$ & $\begin{array}{l}\text { ANOVA } \\
\left(\mathrm{F}_{\mathrm{df}, \mathrm{r}}\right)\end{array}$ \\
\hline $\mathrm{CL}$ & $\begin{array}{c}1.43 \pm 0.14 ; 0.09 \\
(1.56-1.28)\end{array}$ & $\begin{array}{l}0.93 \pm 0.08 ; 0.08 \\
\quad(1.00-0.84)\end{array}$ & $\begin{array}{c}0.79 \pm 0.05 ; 0.07 \\
\quad(0.85-0.72)\end{array}$ & $\begin{array}{l}1.12 \pm 0.08 ; 0.08 \\
\quad(1.25-1.00)\end{array}$ & $\mathrm{F}_{3,15}=32.9 * * *$ \\
\hline Os L & $\begin{array}{c}0.41 \pm 0.09 ; 0.23 \\
\quad(0.40-0.27)\end{array}$ & $\begin{array}{c}0.32 \pm 0.02 ; 0.05 \\
\quad(0.34-0.30)\end{array}$ & $\begin{array}{l}0.24 \pm 0.06 ; 0.24 \\
\quad(0.33-0.20)\end{array}$ & $\begin{array}{c}0.32 \pm 0.06 ; 0.17 \\
\quad(0.40-0.27)\end{array}$ & $\mathrm{F}_{3,15}=4.8^{*}$ \\
\hline Os W & $\begin{array}{c}0.20 \pm 0.00 ; 0.00 \\
(0.20-0.20)\end{array}$ & $\begin{array}{c}0.10 \pm 0.00 ; 0.00 \\
(0.10-0.10)\end{array}$ & $\begin{array}{c}0.12 \pm 0.03 ; 0.26 \\
\quad(0.16-0.09)\end{array}$ & $\begin{array}{l}0.13 \pm 0.03 ; 0.21 \\
\quad(0.15-0.08)\end{array}$ & $\mathrm{F}_{3,15}=11.8 * * *$ \\
\hline PoL & $\begin{array}{c}2.29 \pm 0.20 ; 0.09 \\
\quad(2.44-2.06)\end{array}$ & 1.71 & $\begin{array}{c}1.20 \pm 0.11 ; 0.09 \\
(1.28-1.13)\end{array}$ & $\begin{array}{c}1.66 \pm 0.22 ; 0.13 \\
(2.00-1.40)\end{array}$ & $\mathrm{F}_{3,7}=12.6 * *$ \\
\hline PoW & $\begin{array}{l}0.71 \pm 0.08 ; 0.12 \\
\quad(0.80-0.63)\end{array}$ & 0.5 & $\begin{array}{c}0.35 \pm 0.03 ; 0.10 \\
\quad(0.38-0.33)\end{array}$ & $\begin{array}{l}0.53 \pm 0.06 ; 0.11 \\
\quad(0.57-0.43)\end{array}$ & $\mathrm{F}_{3,7}=12.6 * *$ \\
\hline $\mathrm{AgL}$ & $\begin{array}{c}1.11 \pm 0.13 ; 0.09 \\
(1.23-0.98)\end{array}$ & 0.98 & $\begin{array}{l}0.60 \pm 0.01 ; 0.01 \\
\quad(0.61-0.60)\end{array}$ & $\begin{array}{l}0.75 \pm 0.04 ; 0.06 \\
\quad(0.80-0.68)\end{array}$ & $\mathrm{F}_{3,7}=22.9 * * *$ \\
\hline $\mathrm{CgL}$ & $\begin{array}{c}0.13 \pm 0.05 ; 0.05 \\
\quad(1.17-1.07)\end{array}$ & 0.69 & $\begin{array}{c}0.60 \pm 0.14 ; 0.23 \\
\quad(0.70-0.50)\end{array}$ & $\begin{array}{l}0.93 \pm 0.09 ; 0.10 \\
\quad(1.00-0.77)\end{array}$ & $\mathrm{F}_{3,7}=14.7 * *$ \\
\hline BC L & $\begin{array}{c}0.71 \pm 0.11 ; 0.16 \\
(0.84-0.63)\end{array}$ & 0.4 & $\begin{array}{c}0.38 \pm 0.11 ; 0.27 \\
\quad(0.46-0.31)\end{array}$ & $\begin{array}{l}0.35 \pm 0.07 ; 0.21 \\
\quad(0.42-0.25)\end{array}$ & $\mathrm{F}_{3,7}=10.7 * *$ \\
\hline $\mathrm{BC} W$ & $\begin{array}{c}0.39 \pm 0.04 ; 0.10 \\
(0.43-0.36)\end{array}$ & 0.25 & $\begin{array}{l}0.21 \pm 0.06 ; 0.27 \\
\quad(0.25-0.17)\end{array}$ & $\begin{array}{l}0.21 \pm 0.05 ; 0.23 \\
\quad(0.27-0.15)\end{array}$ & $\mathrm{F}_{3,7}=10.3 * *$ \\
\hline $\mathrm{dBC} L$ & $\begin{array}{c}0.40 \pm 0.05 ; 0.11 \\
\quad(0.43-0.35)\end{array}$ & 0.45 & $\begin{array}{c}0.38 \pm 0.03 ; 0.07 \\
\quad(0.40-0.36)\end{array}$ & $\begin{array}{l}0.46 \pm 0.04 ; 0.10 \\
\quad(0.50-0.40)\end{array}$ & $\mathrm{F}_{3,7}=2^{\mathrm{NS}}$ \\
\hline SR I L & $\begin{array}{c}0.28 \pm 0.03 ; 0.11 \\
(0.31-0.25)\end{array}$ & 0.24 & $\begin{array}{l}0.08 \pm 0.01 ; 0.18 \\
\quad(0.09-0.07)\end{array}$ & $\begin{array}{l}0.26 \pm 0.10 ; 0.41 \\
\quad(0.42-0.15)\end{array}$ & $\mathrm{F}_{3,7}=2.8^{\mathrm{NS}}$ \\
\hline SR II L & absent & absent & absent & $\begin{array}{l}0.16 \pm 0.04 ; 0.26 \\
\quad(0.22-0.12)\end{array}$ & - \\
\hline P L & $\begin{array}{c}1.37 \pm 0.11 ; 0.08 \\
(1.45-1.30)\end{array}$ & $\begin{array}{c}1.00 \pm 0.00 ; 0.00 \\
(1.00-1.00)\end{array}$ & $\begin{array}{l}0.78 \pm 0.12 ; 0.15 \\
\quad(0.87-0.70)\end{array}$ & $\begin{array}{c}1.09 \pm 0.23 ; 0.21 \\
\quad(1.27-0.83)\end{array}$ & $\mathrm{F}_{3,6}=5.5^{*}$ \\
\hline P W & $\begin{array}{c}0.30 \pm 0.00 ; 0.00 \\
(0.30-0.30)\end{array}$ & $\begin{array}{c}0.30 \pm 0.02 ; 0.07 \\
\quad(0.32-0.28)\end{array}$ & $\begin{array}{c}0.17 \pm 0.03 ; 0.20 \\
\quad(0.20-0.15)\end{array}$ & $\begin{array}{l}0.42 \pm 0.06 ; 0.13 \\
\quad(0.47-0.36)\end{array}$ & $\mathrm{F}_{3,6}=17.7 * *$ \\
\hline P L/Head & $\begin{array}{c}1.18 \pm 0.14 ; 0.11 \\
(1.28-1.08)\end{array}$ & $\begin{array}{l}0.90 \pm 0.14 ; 0.16 \\
\quad(1.06-0.80)\end{array}$ & $\begin{array}{l}0.92 \pm 0.11 ; 0.12 \\
\quad(1.00-0.84)\end{array}$ & $\begin{array}{l}0.98 \pm 0.15 ; 0.15 \\
\quad(1.10-0.81)\end{array}$ & $\mathrm{F}_{3,5}=1.8^{\mathrm{NS}}$ \\
\hline ProL & 1.10 & $\begin{array}{c}0.84 \pm 0.11 ; 0.13 \\
\quad(0.94-0.72)\end{array}$ & $\begin{array}{l}0.68 \pm 0.01 ; 0.01 \\
\quad(0.69-0.68)\end{array}$ & $\begin{array}{l}0.76 \pm 0.03 ; 0.04 \\
\quad(0.78-0.72)\end{array}$ & $\mathrm{F}_{3,5}=7.7 *$ \\
\hline ProW & 0.55 & $\begin{array}{l}0.38 \pm 0.04 ; 0.10 \\
\quad(0.42-0.35)\end{array}$ & $\begin{array}{c}0.34 \pm 0.01 ; 0.04 \\
\quad(0.35-0.33)\end{array}$ & $\begin{array}{c}0.37 \pm 0.03 ; 0.08 \\
\quad(0.40-0.34)\end{array}$ & $\mathrm{F}_{3,5}=10.8^{*}$ \\
\hline $\mathrm{RPG}$ & $\begin{array}{c}0.61 \pm 0.03 ; 0.05 \\
(0.64-0.59)\end{array}$ & $\begin{array}{c}0.50 \pm 0.07 ; 0.13 \\
(0.55-0.41)\end{array}$ & $\begin{array}{c}0.52 \pm 0.02 ; 0.05 \\
(0.55-0.49)\end{array}$ & $\begin{array}{c}0.45 \pm 0.03 ; 0.06 \\
(0.46-0.42)\end{array}$ & $\mathrm{F}_{3,10}=11 * * *$ \\
\hline
\end{tabular}




\section{Other localities}

IRAN: Darband stream in Darband village (Azna to Dorud road, ca $16 \mathrm{~km}$ to Azna city), Lorestan Province, $33^{\circ} 25^{\prime} \mathrm{N}, 49^{\circ} 17^{\prime}$ E, ca $1800 \mathrm{~m}$ a.s.1., 23 Jun. 2005.

\section{Description}

Shell ovate-conic with 4-4.5 whorls, height 3-4.5 mm (Fig. 2A-C, Table 2); periostracum yellowish; protoconch approximately $400 \mu \mathrm{m}$ wide with 1.3 whorls and nucleus around $125 \mu \mathrm{m}$ long; protoconch microsculpture wrinkled (Fig. 2D); body whorl about $2 / 3$ of total length; rest of whorls slightly convex with deep sutures; aperture complete, pyriform, often attached to body whorl on the top; thin inner peristome but thicker than outer lip; peristome margin slightly sinuate (Fig. 2C).

Operculum with ca 2 whorls (Fig. 2E-F) and muscle attachment area oval and located near the nucleus.

Radula intermediate length ( $25 \%$ total shell length) bearing about 45 rows of teeth; central tooth formula (5)4-C-4(5)/2-2 (Fig. 2G-H); lateral teeth formula 4-C-4; inner marginal teeth having 18-20 sharp cusps; outer marginal teeth having 15-19 sharp cusps (Fig. 2I).

\section{Pigmentation and anatomy}

Head dark brown pigmented from snout to neck; tentacles brown pigmented except on ocular lobes; snout as long as wide, with medial lobation. Ctenidium extended across most of pallial cavity with 18-21 gill filaments; osphradium two times longer than wide and opposite middle of ctenidium (Fig. $3 \mathrm{~A}$, Table 3).

Nervous system with black pigmentation and elongate (mean RPG ratio 0.60, Table 3); cerebral ganglia equal in size (Fig. 3B).

Female pallial oviduct with a capsule gland longer than albumen gland (Fig. 3C, Table 3); pyriform bursa copulatrix with a duct longer than its length, and lying against the middle section of the albumen gland; renal oviduct white from the insertion point of bursal duct to the seminal receptacle and hereafter black making one or two loops; one elongate seminal receptacle with short duct (Fig. 3D).

Male genitalia with penis gradually tapering with a small distal lobe on inner edge; distal end tapered, and grayish pigmented on the distal section (Fig. 3E-F); prostate gland twice as long as wide (Fig. 3G, Table 3).

\section{Remarks}

This species anatomically resembles I. kermanshahensis, snails of I. zagrosensis, however, are larger and present more cusps in lateral and marginal radular teeth, nervous system with higher RPG ratio, and relatively larger bursa copulatrix and penis.

\section{Ecology and distribution}

Recorded in streams and springs from the Kermanshah and Lorestan provinces (Iran).

Intermaria kermanshahensis (Glöer \& Pešić, 2009) comb. nov.

Figs 4-5, Tables 2-3

Pseudamnicola kermanshahensis Glöer \& Pešić, 2009: 38, pl. 6, figs 7-10.

Sarkhia kermanshahensis - Glöer \& Pešić, 2012: 33, fig. 12h comb. nov. 


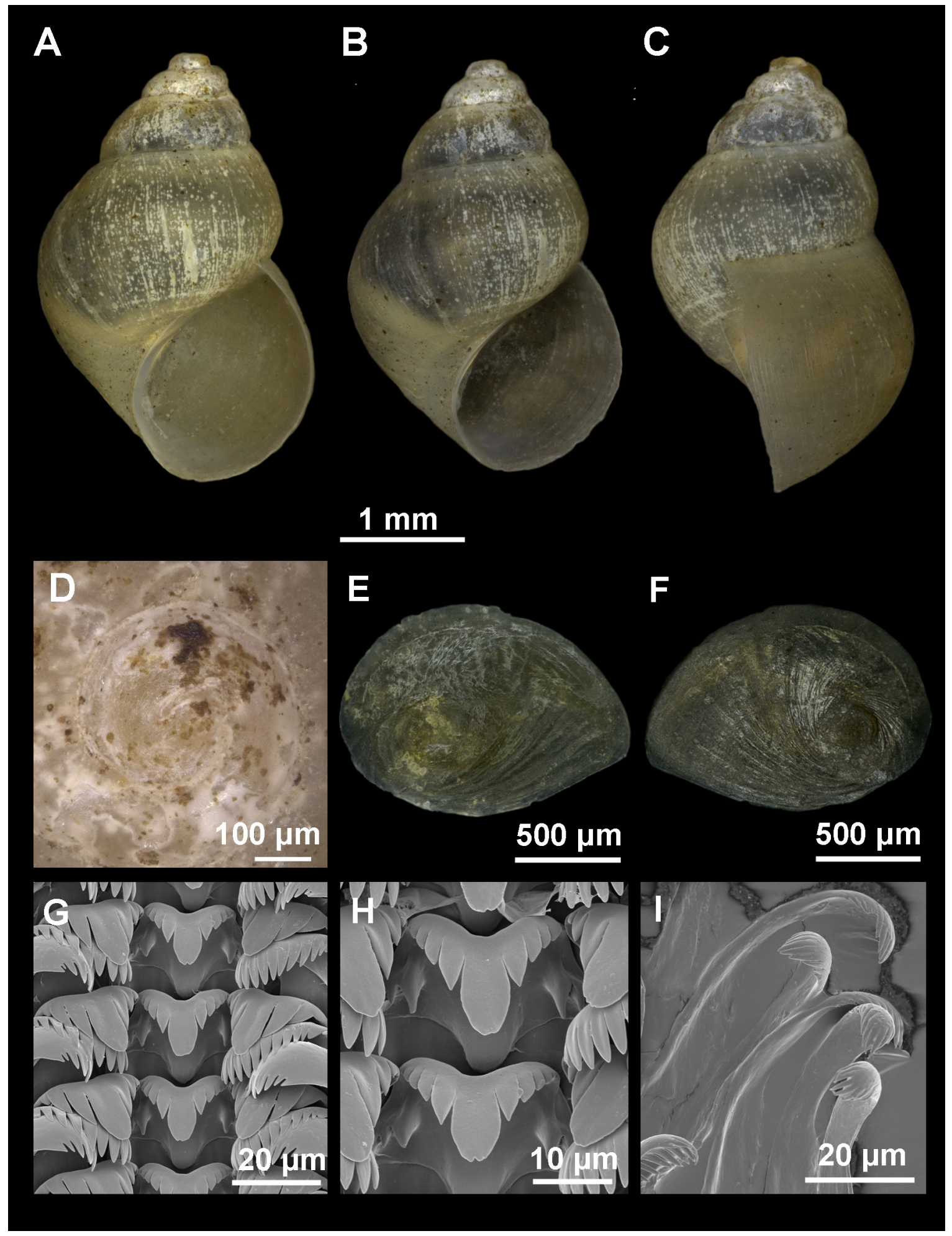

Fig. 4. Shell, operculum and radula of Intermaria kermanshahensis (Glöer \& Pešić, 2009) gen. et comb. nov. from a spring near Sarabe - Sahneh city, Kermanshah Province, Iran. A-B. Shell in front view. C. Shell in lateral view. D. Protoconch and microsculpture. E-F. Internal and external side of the operculum. G. Rows of teeth of the radula. H. Central teeth. I. Detail of outer marginal teeth. 


\section{New diagnosis}

Shell ovate-conic, yellowish, with pyriform aperture; protoconch microsculpture slightly wrinkled; central radular tooth formula 4-C-4/2-2; pyriform bursa copulatrix; one seminal receptacle elongate with short duct; penis gradually tapering with small distal lobe on inner edge, end tapered; nervous system elongated (mean RPG ratio $=0.50$ ) with slight black pigmentation.

\section{Material examined}

\section{Holotype}

IRAN: ZMH 51404: 4 mm height, 2.7 mm width.

\section{Paratypes}

IRAN: ZMH 51405 (5 ex.) and P. Glöer's (66 ex.) collection.

\section{Type locality}

IRAN: spring between Sarab and Sahneh cities, Kermanshah Province, 34²7' N 47 $44^{\prime}$ E, 27 Jun. 2005.

\section{Description}

Shell ovate-conic with 4.25-4.5 whorls, height 3-4 mm (Fig. 4A-C, Table 2); periostracum yellowish; protoconch approximately $500 \mu \mathrm{m}$ wide with 1.4 whorls and nucleus around $135 \mu \mathrm{m}$ long; protoconch microsculpture slightly wrinkled (Fig. 4D); body whorl about $2 / 3$ total length; rest of whorls slightly convex with deep sutures; aperture complete, pyriform, inner lip thicker than outer lip; peristome margin straight (Fig. 4C).

Operculum with 2 whorls approximately (Fig. 4E-F) and muscle attachment area oval and located near the nucleus.

Radula intermediate length ( $25 \%$ total shell length) bearing about 55 rows of teeth; central tooth formula 4-C-4/2-2 (Fig. 4G, H); lateral teeth formula 3-C-3; inner marginal teeth having 15-18 sharp cusps; outer marginal teeth having 12-14 sharp cusps (Fig. 4I).

\section{Pigmentation and anatomy}

Head light brown pigmented from snout to neck; tentacles also brown pigmented except on ocular lobes; snout as long as wide, with medial lobation. Ctenidium extended across most of pallial cavity with 18-21 narrow gill filaments; osphradium three times longer than wide and opposite middle of ctenidium (Fig. 5A, Table 3).

Nervous system with black pigmentation and elongate (mean RPG ratio 0.50); cerebral ganglia equal in size (Fig. 5B, Table 3).

Female pallial oviduct with a capsule gland slightly shorter than albumen gland (Fig. 5C, Table 3); pyriform bursa copulatrix with a duct as long as bursa length, and lying against the middle section of the albumen gland; renal oviduct white from the insertion point of bursal duct to the seminal receptacle and hereafter black making one or two loops; one elongate seminal receptacle with short duct (Fig. 5D).

Male genitalia with penis gradually tapering bearing a small distal lobe in the inner edge; slightly grayish pigmented on the distal section in some specimens (Fig. 5E, F); prostate gland about two times longer than wide (Fig. 5G, Table 3). 


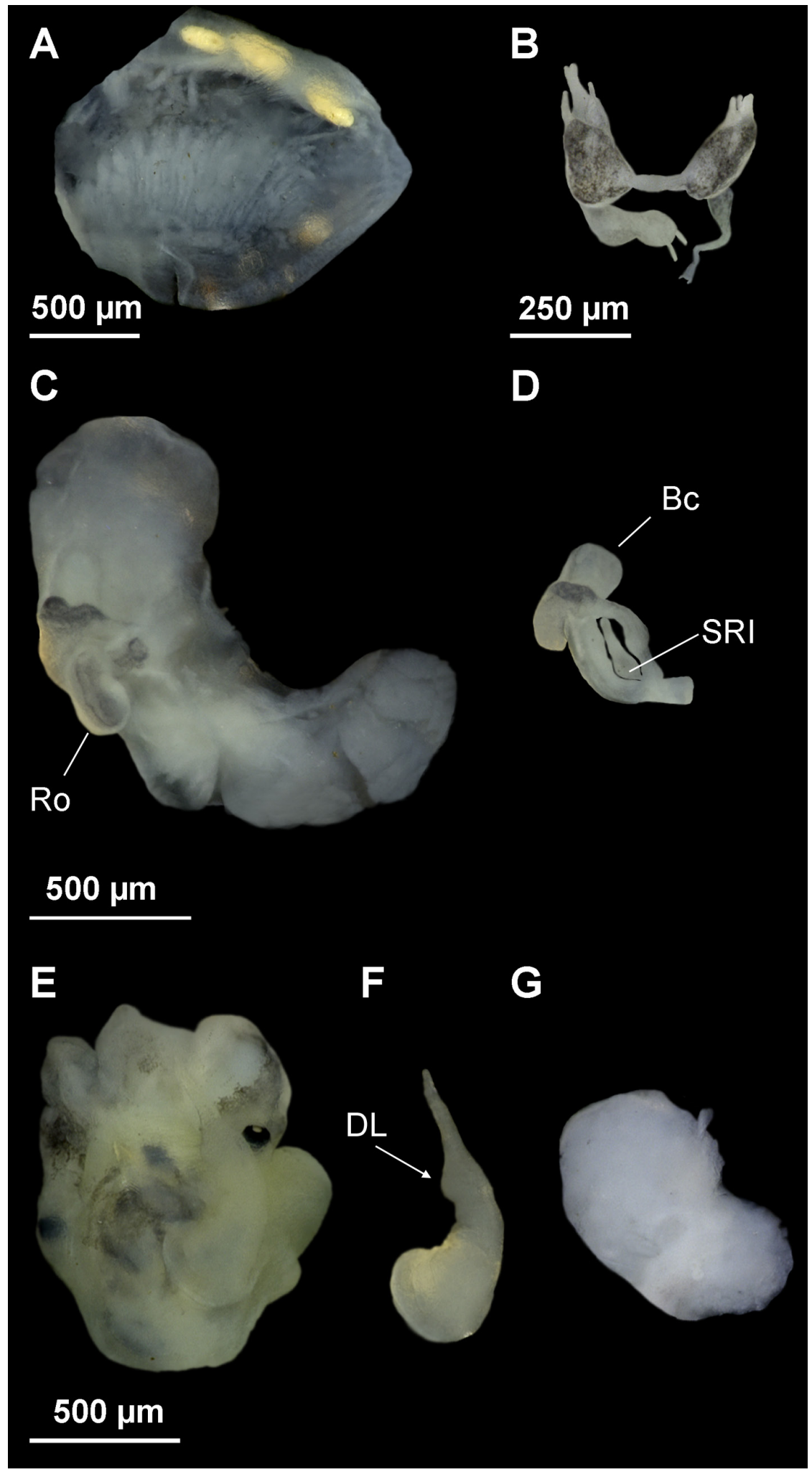

Fig. 5. Anatomy of Intermaria kermanshahensis (Glöer \& Pešić, 2009) gen. et comb. nov. from a spring near Sarabe - Sahneh city, Kermanshah Province, Iran. A. Ctenidium and osphradium. B. Partial nervous system. C. Female genitalia. D. Bursa copulatrix and seminal receptacle. E-F. Head of a male and penis. G. Prostate gland. Anatomical abbreviations given in the Material and methods section, except DL (distal lobe). 


\section{Remarks}

Apart from differences in body dimensions and in certain anatomical features (explained above), the penial distal lobe is more prominent in this species than in I. zagrosensis. Uncorrected genetic distances are, on the contrary, low between these two species $(0.2 \%$ for COI fragment, Table 4$)$, though sister taxa in the subfamily Pyrgulinae are often genetically very close (e.g., COI p-distances of $0.3 \%$ between species of the genus Macedopyrgula Radoman, 1973). Given this minor genetic variation between congeners, these observed anatomical differences could potentially reflect intraspecific variation.

\section{Ecology and distribution}

Known only from a spring in the Kermanshah Province, Iran.

\section{Genus Persipyrgula gen. nov. urn:1sid:zoobank.org:act:6E9922EE-56CD-4BBC-BF4E-9224C75859C0}

\section{Type species}

Pseudamnicola saboori Glöer \& Pešić, 2009.

\section{Diagnosis}

Shell ovate-conic, 2.5 to $4.5 \mathrm{~mm}$ high; large and convex body whorl; rest of the whorls small and slightly convex; aperture complete, pyriform, slightly angled on the top and separated from body whorl. Operculum corneous, yellowish, thin, pliable, ellipsoidal, paucispiral with submarginal nucleus. From one to two pairs of basal cusps in radular central tooth. Ctenidium occupying nearly the entire length of pallial cavity and bearing well-developed gill filaments. Osphradium opposite approximate middle of ctenidium. Bursa copulatrix posterior positioned relative to albumen gland; pigmented renal oviduct; one small pyriform seminal receptacle without duct. Prostate gland bean-shaped, about twice as long as wide; exit of the pallial vas deferens from the posterior-most section of the prostate gland and seminal vesicle entering the prostate gland in its middle section; penis simple, gradually tapering, with a distal end tapered. Nervous system with black pigmentation typically elongated.

\section{Etymology}

Referring to Persia, the historic name of the region where the genus was found, and to Pyrgula, type genus of the subfamily Pyrgulinae.

\section{Remarks}

Though similar in shell features, this genus differs from Pseudamnicola mainly due to its small and pyriform seminal receptacle in the female genitalia (long and elongate in Pseudamnicola), shorter prostate gland, tapered and simple penis (triangular and folded in the latter), and because of the occasional possession of two pairs of basal cusps in the central radular tooth. Differences between Persipyrgula gen. nov. and Intermaria gen. nov. are: shell dimensions (larger in the latter), aperture not fused to body whorl in Persipyrgula gen. nov., one (occasionally two) vs two pairs of basal cusps in the central radular tooth, small pyriform vs elongate seminal receptacle, and absence vs presence of a small distal lobe on the inner edge of the penis, respectively.

Persipyrgula saboori (Glöer \& Pešić, 2009) comb. nov.

Figs 6-7, Tables 2-3

Pseudamnicola saboori Glöer \& Pešić, 2009: 36, pl. 6, figs 1-3. 


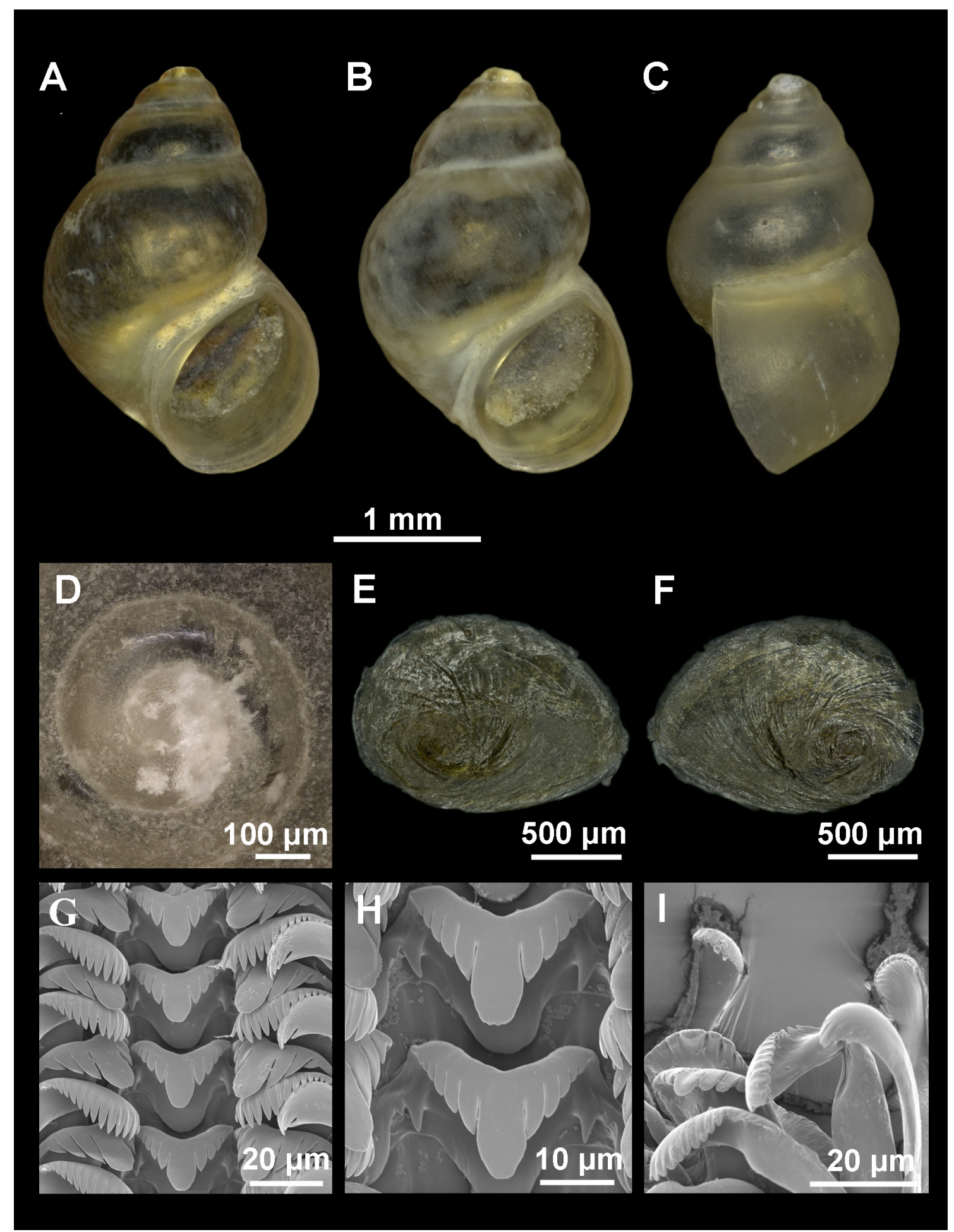

Fig. 6. Shell, operculum and radula of Persipyrgula saboori (Glöer \& Pešić, 2009) gen. et comb. nov. from Zou Eram spring in Zou Eram village, Khorrasan Province, Iran. A-B. Shell in front view. C. Shell in lateral view. D. Protoconch and microsculpture. E-F. Internal and external side of the operculum. G. Rows of teeth of the radula. H. Central teeth. I. Detail of outer marginal teeth. 


\section{New diagnosis}

Shell ovate-conic, yellowish, with pyriform aperture; protoconch microsculpture wrinkled; central radular tooth formula 5-C-5/(2)1-1(2); pyriform bursa copulatrix, with a duct longer than bursa; one small pyriform seminal receptacle with no distinct duct; penis gradually tapering and simple, with distal end tapered and grayish pigmented; nervous system elongated (mean RPG ratio $=0.52$ ) with slight black pigmentation.

\section{Material examined}

\section{Holotype}

IRAN: ZMH 51402: $4.4 \mathrm{~mm}$ height, $3.0 \mathrm{~mm}$ width.

\section{Paratypes}

IRAN: ZMH 51403 (5 ex.) and P. Glöer's (46 ex.) collection.

\section{Type locality}

IRAN: Zou Eram spring in Zou Eram village, Shirvan city, Khorasan Province, $37^{\circ} 20^{\prime} \mathrm{N}, 57^{\circ} 40^{\prime} \mathrm{E}$, ca 1600 m, 11 Jun. 2005.

\section{Description}

Shell ovate-conic with 4-4.5 whorls, height 2.5-4.4 mm (Fig. 6A-C, Table 2); periostracum yellowish; protoconch approximately $350 \mu \mathrm{m}$ wide with 1.25 whorls and nucleus around $120 \mu \mathrm{m}$ long; protoconch microsculpture wrinkled, more intense on apex (Fig. 6D); body whorl about $2 / 3$ total length; rest of whorls slightly convex with a deep suture; aperture complete and pyriform; inner lip thicker than outer lip; peristome margin straight (Fig. 6C).

Operculum with 2.5 whorls approximately (Fig. 6E-F) and muscle attachment area oval located near the nucleus.

Radula intermediate length ( $30 \%$ total shell length) bearing about 50 rows of teeth; central tooth formula 5-C-5/(2)1-1(2) (Fig. 6G-H); lateral teeth formula 3-C-3; inner marginal teeth having 15-18 cusps; outer marginal teeth having 14-16 cusps (Fig. 6I).

\section{Pigmentation and anatomy}

Head dark brown pigmented from snout to the penial base; tentacles also brown pigmented except on ocular lobes; snout as long as wide, with medial lobation. Ctenidium in middle region of pallial cavity with 18-20 narrow gill filaments; osphradium two times longer than wide and opposite middle of ctenidium (Fig. 7A, Table 3).

Nervous system with black pigmentation and elongate (mean RPG ratio 0.52, Table 3); cerebral ganglia equal in size (Fig. 7B).

Female pallial oviduct with a capsule gland and albumen gland similar in size (Fig. 7C, Table 3); pyriform bursa copulatrix with a duct about $50-100 \%$ of bursa length; renal oviduct white straight from the insertion point of bursal duct to where it begins to fold; hereafter black pigmented making a simple loop; one small pyriform seminal receptacle with no distinct duct (Fig. 7D).

Male genitalia with penis simple, gradually tapering, with distal end tapered grayish pigmented (Fig. 7EF); prostate gland bean-shaped, about two times longer than wide (Fig. 7G, Table 3). 

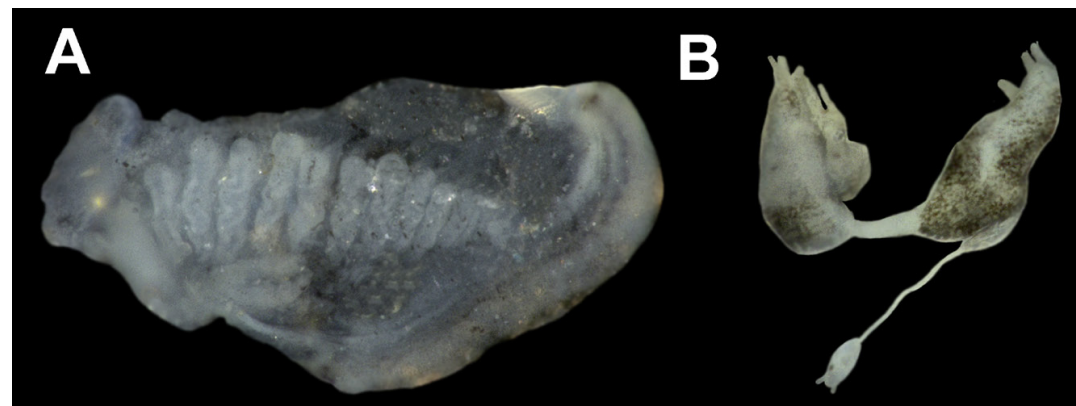

\section{$500 \mu \mathrm{m}$}

\section{0 um}

C

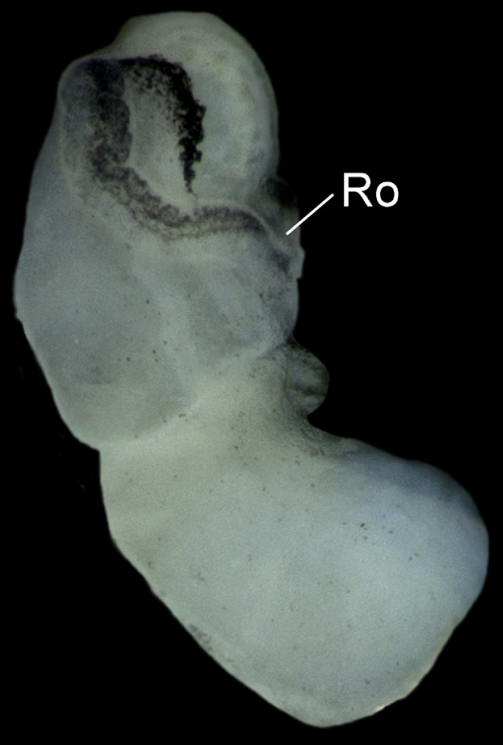

D

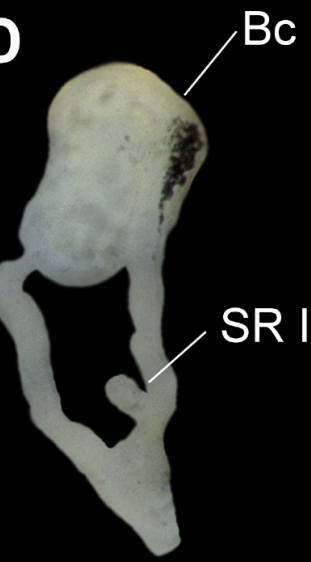

\section{$500 \mu \mathrm{m}$}

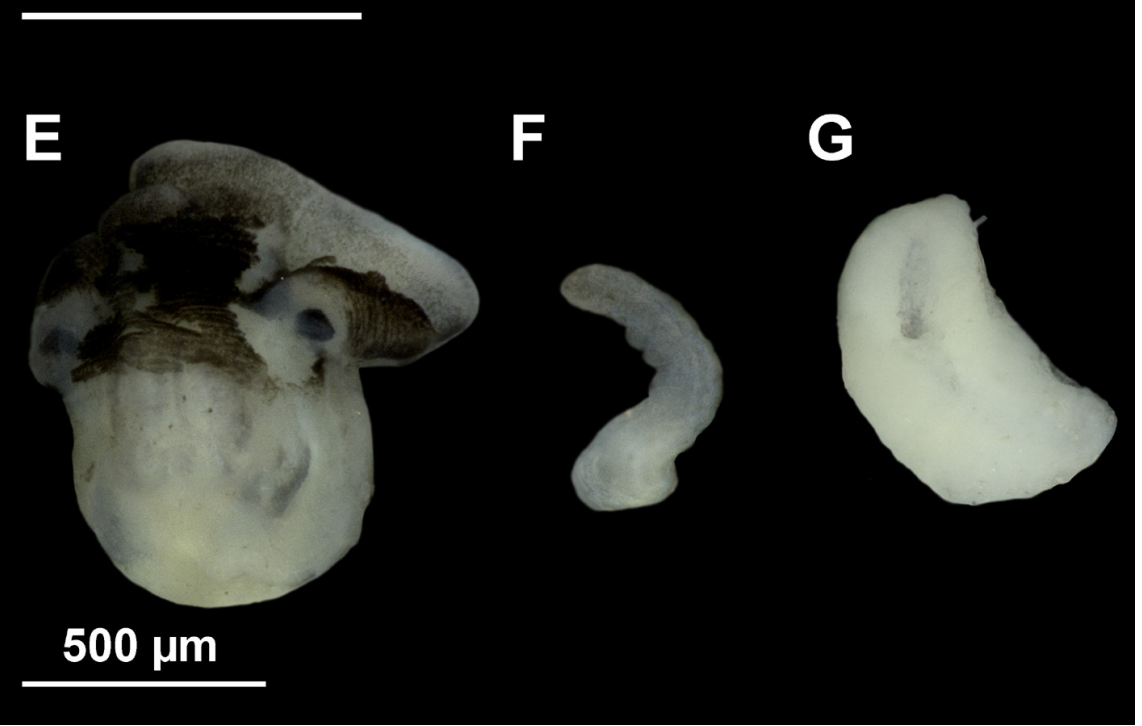

Fig. 7. Anatomy of Persipyrgula saboori (Glöer \& Pešić, 2009) gen. et comb. nov. from Zou Eram spring in Zou Eram village, Khorrasan Province, Iran. A. Ctenidium and osphradium. B. Partial nervous system. C. Female genitalia. D. Bursa copulatrix and seminal receptacle. E-F. Head of a male and penis. G. Prostate gland. Anatomical abbreviations given in the Material and methods section. 


\section{Remarks}

The small pyriform seminal receptacle and the posterior position of bursa copulatrix relative to albumen gland are the exceptional features of this species related to the species comprising the new pyrgulinid lineage found in this study. Moreover, P. saboori is the most distantly related taxa of this newly recovered lineage (COI p-distances from 6.5 to $8.5 \%$, Table 4).

\section{Ecology and distribution}

Only recorded in Khorasan and Markazi provinces (Iran).

Genus Shadinia Akramowski, 1976

\section{Type species}

Pyrgula terpoghassiani Shadin, 1952.

\section{New diagnosis}

Shell ovate-conic, 3 to $5 \mathrm{~mm}$ high; large and convex body whorl; rest of the whorls tall and convex; aperture complete, pyriform, angled on the top and fused to the body whorl. Operculum corneous, yellowish, thin, pliable, ellipsoidal, paucispiral with submarginal nucleus. From two to three pairs of basal cusps in radular central tooth. Ctenidium occupying nearly the entire length of pallial cavity and bearing well-developed gill filaments. Osphradium opposite approximate middle of ctenidium. Bursa copulatrix lying against the middle section of the albumen gland; pigmented renal oviduct; two opposite seminal receptacles, SRII smaller than SRI. Prostate gland bean-shaped, about twice as long as wide; exit of the pallial vas deferens from the posterior-most section of the prostate gland and seminal vesicle entering the prostate gland in its middle section; penis simple, gradually tapering, with a distal end tapered dark pigmented. Nervous system with black pigmentation and moderately concentrated.

Shadinia terpoghassiani (Shadin, 1952)

Figs 8-9; Tables 2-3

Pyrgula terpoghassiani Shadin, 1952: 227

Pyrgula terpoghassiani-Akramowski 1952, nom. nud.

\section{New diagnosis}

Shell ovate-conic, yellowish, with two weak parallel keels along the body whorl; pyriform aperture; protoconch microsculpture wrinkled; central radular tooth formula 3-C-3/(2)3-3(2); pyriform bursa copulatrix; SRI elongate with a short duct and SRII smaller, globular and without duct; penis gradually tapering and simple, with a distal end tapered and black pigmented; nervous system moderately concentrated (mean $\mathrm{RPG}$ ratio $=0.45$ ) and with slight black pigmentation.

\section{Material examined}

Type material not specified.

\section{Type locality}

ARMENIA: Lake Aiger-Lich, south of Metzamor, Armavia province, $40^{\circ} 08.573^{\prime} \mathrm{N}, 44^{\circ} 10.270^{\prime} \mathrm{E}$, 18 May 2008. 


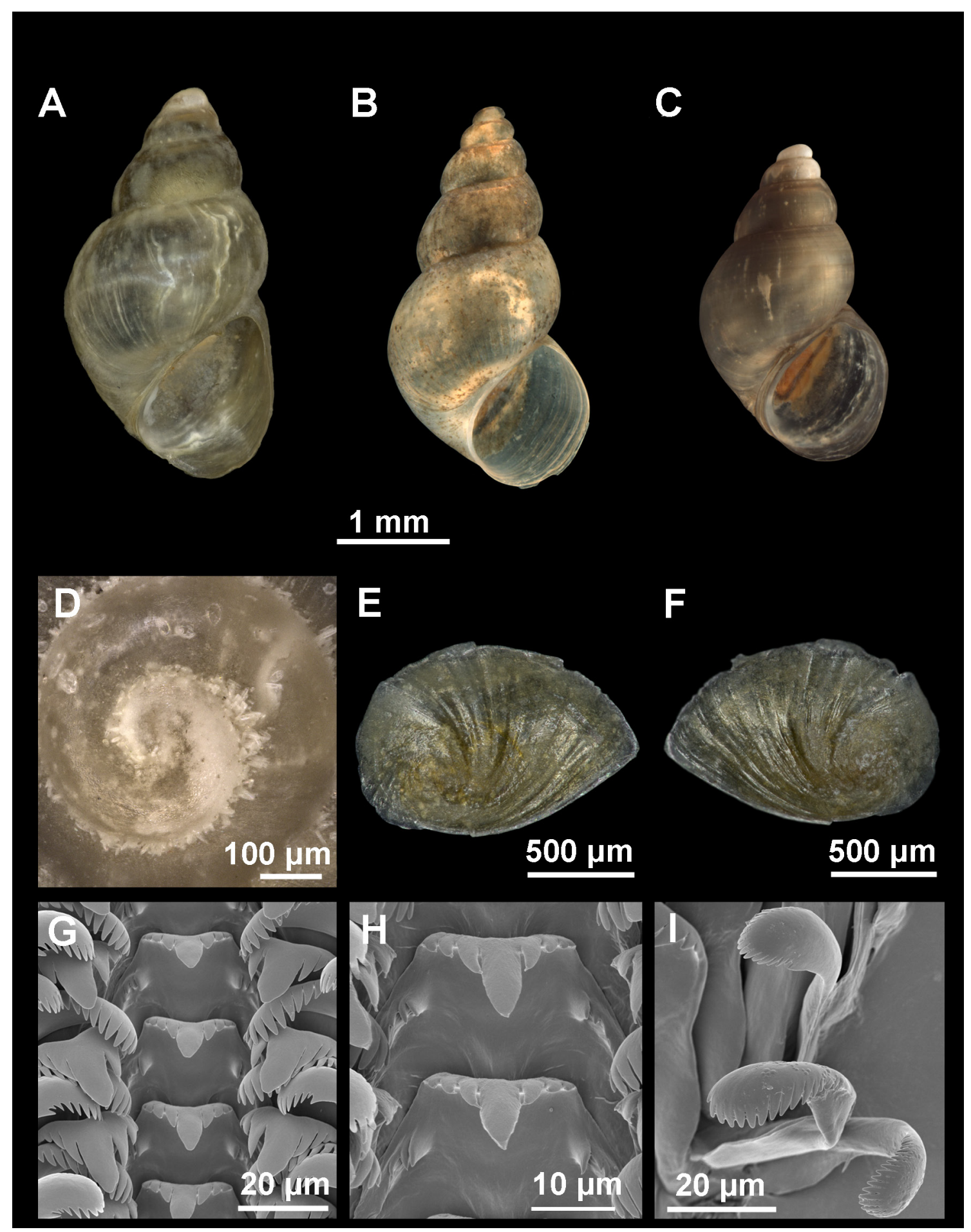

Fig. 8. Shell, operculum and radula of Shadinia terpoghassiani (Shadin, 1952) from Lake Aiger-Lich, Armenia. A-C. Shell in front view. D. Protoconch and microsculpture. E-F. Internal and external side of the operculum. G. Rows of teeth of the radula. H. Central teeth. I. Detail of outer marginal teeth. 


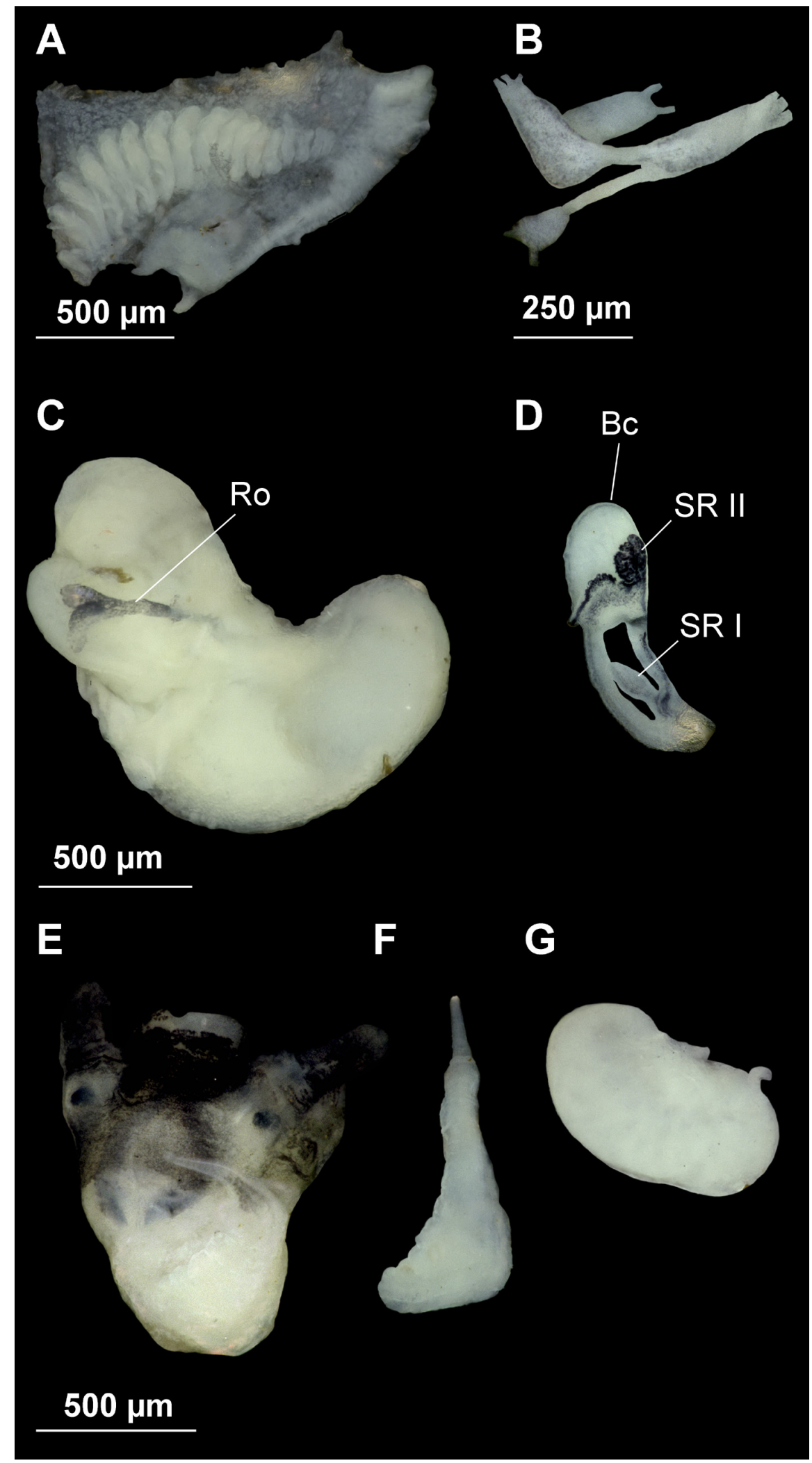

Fig. 9. Anatomy of Shadinia terpoghassiani (Shadin, 1952) from Lake Aiger-Lich, Armenia. A. Ctenidium and osphradium. B. Partial nervous system. C. Female genitalia. D. Bursa copulatrix and seminal receptacle. E-F. Head of a male and penis. G. Prostate gland. Anatomical abbreviations given in the Material and methods section. 


\section{Description}

Shell ovate-conic with 4-4.5 whorls, height 2.6-3.6 mm (Fig. 8A-C, Table 2); periostracum yellowish; protoconch approximately $385 \mu \mathrm{m}$ wide with 1.3 whorls and nucleus around $125 \mu \mathrm{m}$ long; protoconch microsculpture wrinkled (Fig. 8D); body whorl about $2 / 3$ total length and bearing two weak spiral keels; rest of whorls tall and convex with a deep suture; aperture complete, pyriform, with an inner lip thicker than outer lip; peristome margin slightly sinuate.

Operculum with 2.5 whorls approximately (Fig. 8E-F) and muscle attachment area oval and located near the nucleus.

Radula intermediate length (25\% total shell length) bearing around 45 rows of teeth; central tooth formula 3-C-3/(2)3-3(2) (Fig. 8G-H); lateral teeth formula 3-C-3; inner and outer marginal teeth bearing 17-21 and 19-25 cusps, respectively (Fig. 8I).

\section{Pigmentation and anatomy}

Head dark brown pigmented from snout to penial base; pigmentation clearer on neck; tentacles also brown pigmented except on ocular lobes; snout as long as wide, with medial lobation. Ctenidium in middle region of pallial cavity with 18-20 gill filaments; osphradium two to three times longer than wide and opposite middle of ctenidium (Fig. 9A, Table 3).

Nervous system with black pigmentation and moderately concentrated (mean RPG ratio 0.45 , Table 3); cerebral ganglia equal in size (Fig. 9B).

Female genitalia with a capsule gland longer than albumen gland (Fig. 9C, Table 3); pyriform bursa copulatrix lying against the middle section of the albumen gland; bursal duct longer than bursa length; renal oviduct straight and white from the insertion point of bursal duct to SRII; hereafter black pigmented making one or two loops; two opposite seminal receptacles; SRI pyriform with short duct and SRII smaller, black pigmented, globular and sessile (Fig. 9D).

Male genitalia bearing a penis simple, gradually tapering, with a distal end tapered, and black pigmented on the distal section (Fig. 9E-F); bean-shaped prostate gland about two times longer than wide (Fig. 9G, Table 3).

\section{Remarks}

Specimens from the type locality varied in shell dimensions and whorl convexity (Fig. 8A-C and Shadin 1952: fig. 51), though they were similar in their anatomical features. So far, Shadinia is the only pyrgulinid genus bearing two seminal receptacles (for a morphological review of the Pyrgulinae subfamily see Radoman (1983); the anatomy of Pyrgula Cristofori \& Jan, 1832 and Dianella Gude, 1913 is described in Szarowska 2006). Size of seminal receptacles varies slightly between the three anatomically known species, being smaller in S. terpoghassiani than in S. bjniensis. Moreover, some specimens of $S$. terpoghassiani are larger (shell height $S$. bjniensis $3.6-4.0 \mathrm{~mm}$, S. terpoghassiani $5.2 \mathrm{~mm}$, see Shadin 1952) and often two weak spiral keels are present in the shell body whorl. Three basal cusps in the central radular tooth are also present in S. akramowskii (Shadin 1952). The species of Shadinia here analyzed differ from each other by $0.4 \%$ COI p-distances (Table 4 ).

\section{Ecology and distribution}

Armenia and Nakhchivan province of Azerbaijan (Akramowski 1976). 
Table 4. Uncorrected COI p-distances between species of the subfamilies Pyrgulinae and Pseudamnicolinae.

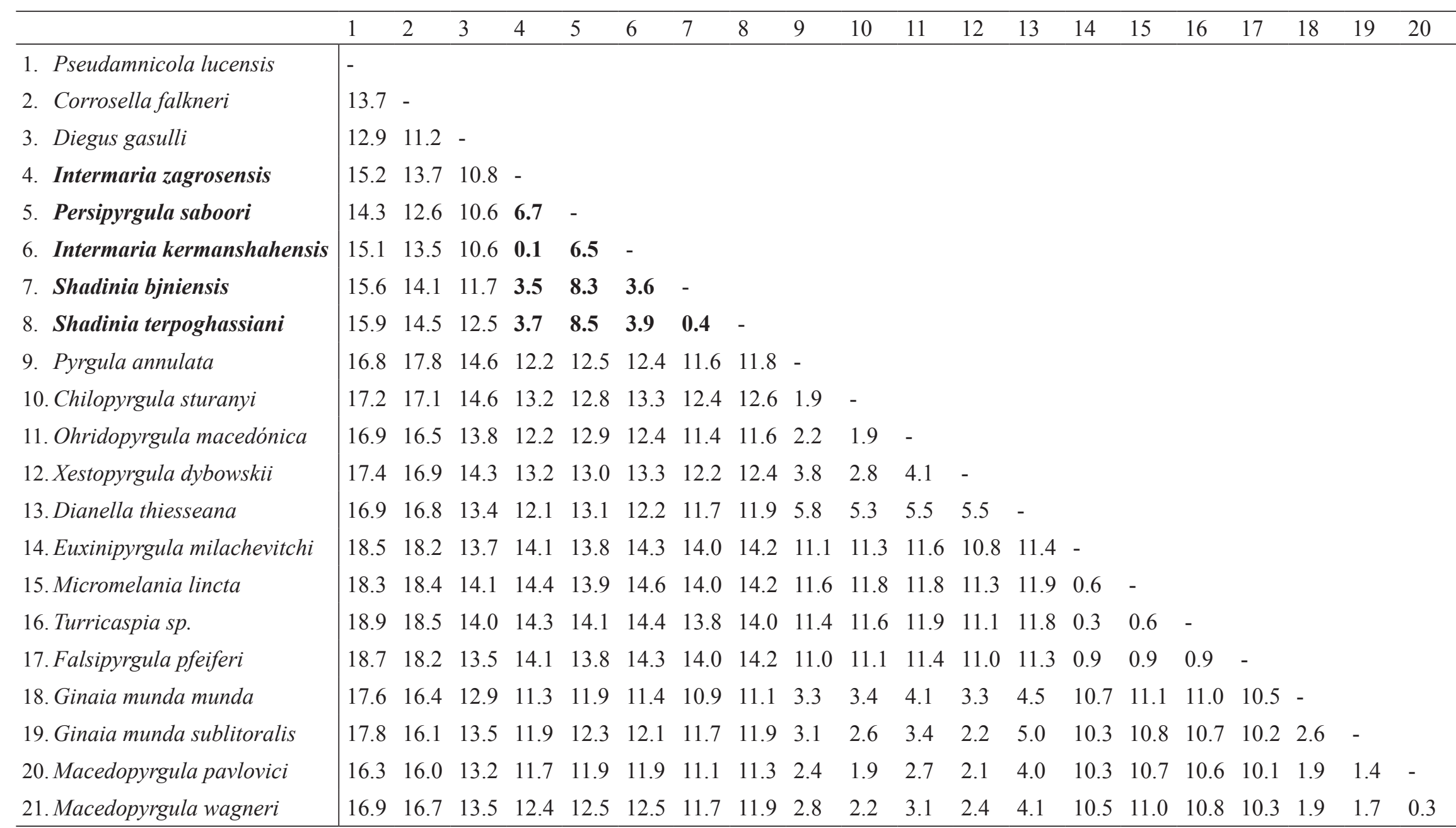




\section{Discussion}

Our results were consistent with our proposed hypothesis of the incorrect assignment of the Pseudamnicola species from Iran described by Glöer \& Pešić (2009). Thus, molecular and morphological analyses revealed that shell shape and penis morphology alone are not sufficiently informative for genus assignment. Our phylogenetic data recovered those Iranian species and the genus Shadinia as a potential monophyletic group appearing outside Pseudamnicola and the subfamily Pseudamnicolinae (Fig. 1). A sister relationship between this new lineage and other pyrgulinid species was identified (despite the limited resolution of COI for inferring phylogenetic relationships on the subfamily level). However, p-distances between the Ponto-Caspian genera and the molecularly analyzed Pseudamnicolinae and Pyrgulinae taxa proved similar (13.6\% and $13 \%$, respectively). In effect, our phylogenetic reconstruction suggests these new taxa may comprise a different subfamily intermediate between the other two. Notwithstanding, this would need confirmation through more comprehensive sampling of the area and examining other potential new representatives of this lineage. As more shared synapomorphies with the pyrgulinid group were detected (such as a tapered penis, zero to two seminal receptacles, zero to three pairs of basal cusps in the central radular tooth and spiral keels on the shell, see Radoman 1955, 1983) than with Pseudamnicola (diagnosed by, one elongate seminal receptacle, one pair of basal cusps in the central radular tooth, triangular penis with folds and simple shells slightly longer than wide, of which the latter two are not present in Pyrgulinae, see Boeters 1988; Szarowska et al. 2009; Delicado et al. 2014), we tentatively consider these taxa as belonging to the subfamily Pyrgulinae.

Despite the low p-distances characterizing these Ponto-Caspian species, morphological evidence led us to consider them as different genera, here described as Intermaria gen. nov. and Persipyrgula gen. nov. This pattern of high morphological variability between closely related genera has been also observed in other pyrgulinid groups (see Wilke et al. 2007). In addition, the discovery of these genera suggests that other species from the Ponto-Caspian region assigned to Pseudamnicola according to shell shape and penis morphology could effectively be members of this newly discovered lineage or even constitute other clades. For instance, P. kayseriensis Glöer, Yıldırım \& Kebapçi, 2015, P. gullei Glöer, Yıldırım \& Kebapçi, 2015 and P. vinarskii Glöer \& Georgiev, 2012 from Turkey bear similar shell and penis morphologies as the genera here described, and despite a more conical shell, the species

A

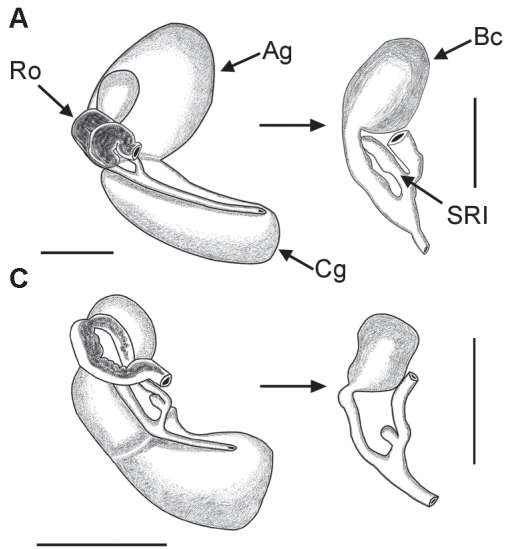

B

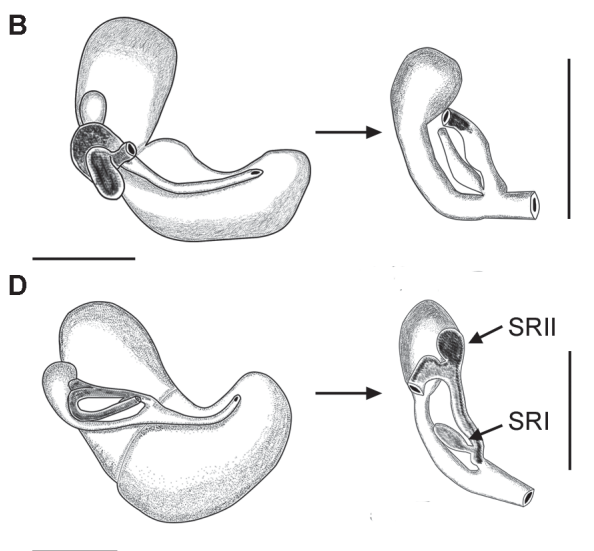

Fig. 10. Distal female genitalia and associated structures of the Ponto-Caspian species. A. Intermaria zagrosensis (Glöer \& Pešić, 2009) gen. et comb. nov. B. Intermaria kermanshahensis (Glöer \& Pešić, 2009) gen. et comb. nov. C. Persipyrgula saboori (Glöer \& Pešić, 2009) gen. et comb. nov. D. Shadinia terpoghassiani (Shadin, 1952). Anatomical abbreviations given in the Material and method section. Scale bars: $500 \mu \mathrm{m}$. 
Sarkhia sarabensis Glöer \& Pešić, 2012 from Iran also shows similar penis characteristics. Additional anatomical descriptions and phylogenetic data might soon clarify this issue.

In conclusion, our morphological and molecular data question the species richness of the genus Pseudamnicola, and indicate that the morphological characters traditionally used for the recognition of Ponto-Caspian hydrobiid species (i.e., conchyliologic) need to be complemented with other features mainly related to their genital and trophic systems. The high morphological disparity observed among closely related pyrgulinid genera calls for an intensive study of the reason why some hydrobiids are so similar and others so diverse. Our phylogeny identified a new potential lineage that could represent a new subfamily between Pseudamnicolinae and Pyrgulinidae, thus contributing to the knowledge of evolutionary patterns in the family Hydrobiidae. Our findings also provide future direction for research on the biodiversity, systematics and biogeography of hydrobiid gastropods, particularly those from the Ponto-Caspian region.

\section{Acknowledgements}

The authors thank the German Academic Exchange Service (DAAD) for enabling T. Hauffe and S. Sereda to collect the material of Shadinia in Armenia, and Barbara Hoenig and Sabine Agel (Imaging Unit, Biomedical Research Centre Seltersberg, Justus Liebig University, Giessen) for their assistance with the ESEM photomicrographs. The English was reviewed by A. Burton. Two anonymous reviewers contributed to the improvement of this manuscript. This study was supported by a fellowship of the postdoctoral program Just'us (Junior Science and Teaching Units, Justus Liebig University, Giessen) granted to D.D.

\section{References}

Akaike H. 1974. A new look at the statistical model identification. IEEE Transactions on Automatic Control 19: 716-723. http://dx.doi.org/10.1109/TAC.1974.1100705

Akramowski N.N. 1952. O nakhozhdenii sovremennogo predstavitelya roda Pyrgula Cristof. et Jan v doline srednego techeniya Araksa (Gastropoda - Prosobranchia, Hydrobiidae). Akademiya Nauk Armyanskoj SSR 84: 631-632.

Akramovski N.N. 1976. Fauna of Armenian SSR: Mollusks (Mollusca). Akademiya Nauk Armyanskoj SSR, Armenia.

Arconada B. \& Ramos M.A. 2003. The Ibero-Balearic region: one of the areas of highest Hydrobiidae (Gastropoda, Prosobranchia, Rissooidea) diversity in Europe. Graellsia 59: 91-104.

Badzoshvili T.I. 1979. Mollyuski meotisa zapadnoy Gruzii. Metsniereba, Tbilisi.

Boeters H.D. 1988. Moitessieriidae und Hydrobiidae in Spanien und Portugal. Archiv für Molluskenkunde 118: 181-261.

Darriba D., Taboada G.L., Doallo R. \& Posada D. 2012. jModelTest 2: more models, new heuristics and parallel computing. Nature Methods 9: 772-772. http://dx.doi.org/10.1038/nmeth.2109

Davis G.M., Chen C.E., Wu C., Kuang T.F., Xing X.G., Li L., Liu W.J. \& Yan Y.L. 1992. The Pomatiopsidae of Hunan, China (Gastropoda: Rissoacea). Malacologia 34: 143-342. Available from http://biodiversitylibrary.org/page/13129606 [accessed 30 May 2016]

Davis G.M., Guo Y.H., Hoagland K.E., Chen P.L., Zheng L.C., Yang H.M., Chen D.J. \& Zhou Y.F. 1986. Anatomy and Systematics of Triculini (Prosobranchia: Pomatiopsidae: Triculinae), freshwater snails from Yunnan, China, with descriptions of new species. Proceedings of the Academy of Natural Sciences of Philadelphia 138: 466-575. 
DELICADO D. et al., New hydrobiid genera from the Ponto-Caspian region

Davis G.M., Kitikoon V. \& Temcharoen P. 1976. Monograph on 'Lithoglyphosis' aperta, the snail of Mekong river Schistosomiasis. Malacologia 15: 241-287. Available from http://biodiversitylibrary.org/ page/13122908 [accessed 30 May 2016]

Davis G.M., Kuo Y.H., Hoagland K.E., Chen P.L., Yang H.M. \& Chen D.J. 1984. Kunmingia, a new genus of Triculinae (Gastropoda: Pomatiopsidae) from China: Phenetic and cladistic relationships. Proceedings of the Academy of Natural Sciences of Philadelphia 136: 165-193.

Delicado D., Machordom A. \& Ramos M.A. 2012. Underestimated diversity of hydrobiid snails. The case of Pseudamnicola (Corrosella) (Mollusca: Caenogastropoda: Hydrobiidae). Journal of Natural History 46: 25-89. http://dx.doi.org/10.1080/00222933.2011.623358

Delicado D., Machordom A. \& Ramos M.A. 2014. Vicariant versus dispersal processes in the settlement of Pseudamnicola (Caenogastropoda, Hydrobiidae) in the Mediterranean Balearic Islands. Zoological Journal of the Linnean Society 171: 38-71. http://dx.doi.org/10.1111/zoj.12124

Delicado D., Machordom A. \& Ramos M.A. 2015. Effects of habitat transition on the evolutionary patterns of the microgastropod genus Pseudamnicola (Mollusca, Hydrobiidae). Zoologica Scripta 44: 403-417. http://dx.doi.org/10.1111/zsc.12104

Delicado D. \& Ramos M.A. 2012. Morphological and molecular evidence for cryptic species of springsnails [genus Pseudamnicola (Corrosella) (Mollusca, Caenogastropoda, Hydrobiidae)]. ZooKeys 190: 55-79. http://dx.doi.org/10.3897/zookeys.190.2555

Egorov R. 2006. Treasure of Russian shells, Supplement 4. Illustrated Catalogue with Selected Identification Keys of the Recent Fresh- and Brackish-water Pectinibranch Molluscs (Gastropoda: Pectinibranchia) of Russia and adjacent regions. Colus, Moscow.

Felsenstein J. 1985. Confidence limits on phylogenies: An approach using the bootstrap. Evolution 39: 783. http://dx.doi.org/10.2307/2408678

Folmer O., Black M., Hoeh W., Lutz R. \& Vrijenhoek R. 1994. DNA primers for amplification of mitochondrial cytochrome c oxidase subunit I from diverse metazoan invertebrates. Molecular marine biology and biotechnology 3: 294-299.

Glöer P., Bößneck U., Walther F. \& Neiber M.T. 2016. New taxa of freshwater molluscs from Armenia (Caenogastropoda: Truncatelloidea: Hydrobiidae). Folia Malacologica 24 (1): 3-8.

Glöer P. \& Georgiev D. 2012. Three new gastropod species from Greece and Turkey (Mollusca: Gastropoda: Rissooidea) with notes on the anatomy of Bythinella charpentieri cabirius Reischütz 1988. North-western journal of Zoology 8: 278-282.

Glöer P., Gürlek M. \& Kara C. 2014. New Pseudamnicola species of Turkey (Mollusca: Gastropoda: Hydrobiidae). Ecologica Montenegrina 1: 103-108.

Glöer P. \& Pešić V. 2009. New freshwater gastropod species of the Iran (Gastropoda: Stenothyridae, Bithyniidae, Hydrobiidae). Mollusca 27: 33-39.

Glöer P. \& Pešić V. 2012. The freshwater snails (Gastropoda) of Iran, with descriptions of two new genera and eight new species. ZooKeys 219: 11-61. http://dx.doi.org/10.3897/zookeys.219.3406

Glöer P., Yıldırım M.Z. \& Kebapçi Ü. 2015. Description of two new species of Pseudamnicola from southern Turkey (Mollusca: Gastropoda: Hydrobiidae). Zoology in the Middle East 61: 139-143. http:// dx.doi.org/10.1080/09397140.2015.1008189

Guindon S. \& Gascuel O. 2003. A simple, fast, and accurate algorithm to estimate large phylogenies by maximum likelihood. Systematic biology 52: 696-704. 
Hershler R. \& Ponder W.F. 1998. A review of morphological characters of hydrobioid snails. Smithsonian Contributions to Zoology 600, Smithsonian Institution, Washington.

Huelsenbeck J.P. 2000. MrBayes: Bayesian Inference of Phylogeny. NCBI, New York.

Huelsenbeck J.P. \& Ronquist F. 2001. MRBAYES: Bayesian inference of phylogenetic trees. Bioinformatics 17: 754-755. http://dx.doi.org/10.1093/bioinformatics/btg180

Hurvich C.M. \& Tsai C.L. 1989. Regression and time series model selection in small samples. Biometrika 76: 297-307.

Kelley K. \& Lai K. 2011. MBESS 3.0.4 (or higher): [computer software and manual]. Available from http://www.cran.r-project.org/ [accessed 30 May 2016]

Liu H.P., Hershler R. \& Clift K. 2003. Mitochondrial DNA sequences reveal extensive cryptic diversity within a western American springsnail. Molecular Ecology 12: 2771-2782. http://dx.doi.org/10.1046/ j.1365-294X.2003.01949.X

R Development Core Team. 2011. R: A Language and Environment for Statistical Computing. Vienna, Austria: R Foundation for Statistical Computing.

Radoman P. 1955. Recherches morphologiques et systematiques sur les Hydrobiides du Lac d'Ohrid. Posebna Izd. Srpska Biol Dr 1: 1-106.

Radoman P. 1983. Hydrobioidea a Superfamily of Prosobranchia (Gastropoda): Systematics. Serbian Academy of Sciences and Arts, Belgrade.

Rambaut A. \& Drummond A. 2009. Tracer v. 1.5. Available from http://tree.bio.ed.ac.uk/software/ tracer/ [accessed 30 May 2016]

Ramos M.A., Arconada B., Moreno D. \& Rolán E. 2000. A new genus and a new species of Hydrobiid snail (Mollusca: Gastropoda: Hydrobiidae) from eastern Spain. Malacologia 42: 75-101. http:// biodiversitylibrary.org/page/12937820

Schreiber K., Hauffe T., Albrecht C. \& Wilke T. 2012. The role of barriers and gradients in differentiation processes of pyrgulinid microgastropods of Lake Ohrid. Hydrobiologia 682: 61-73. http://dx.doi.org/10. 1007/s10750-011-0864-4

Shadin V.I. 1952. Mollusks of fresh and brackish waters of the USSR. Academy of Sciciences of the U.S.S.R., Moscow.

Strong E.E., Gargominy O., Ponder W.F. \& Bouchet P. 2008. Global diversity of gastropods (Gastropoda; Mollusca) in freshwater. In: Balian E.V., Lévêque C., Segers H., Martens K. (eds) Freshwater Animal Diversity Assessment. Developments in Hydrobiology 595 (1): 149-166. http://dx.doi.org/10.1007/ $\underline{\text { s10750-007-9012-6 }}$

Sugiura N. 1978. Further analysis of the data by Akaike's information criterion and the finite corrections. Communications in Statistics - Theory and Methods A 7: 13-26. http://dx.doi. org/10.1080/03610927808827599

Swofford D.L. 2002. PAUP*. Phylogenetic analysis using parsimony (*and other methods). Sinauer Associates, Sunderland, Massachusetts.

Szarowska M. 2006. Molecular phylogeny, systematics and morphological character evolution in the Balkan Rissooidea (Caenogastropoda). Folia Malacologica 14: 99-168. http://dx.doi.org/10.12657/ folmal.014.014

Szarowska M., Grzmil P. \& Falniowski A. 2009. Pseudamnicola Paulucci, 1878 (Gastropoda: Hydrobiidae) in the Balkans. Folia Malacologica 14: 179-190. http://dx.doi.org/10.12657/folmal.014.016 
DELICADO D. et al., New hydrobiid genera from the Ponto-Caspian region

Tamura K. \& Nei M. 1993. Estimation of the number of nucleotide substitutions in the control region of mitochondrial DNA in humans and chimpanzees. Molecular Biology and Evolution 10: 512-526.

Wilke T. 2003. Salenthydrobia gen. nov. (Rissooidea: Hydrobiidae): a potential relict of the Messinian salinity crisis. Zoological Journal of the Linnean Society 137: 319-336. http://dx.doi.org/10.1046/ j.1096-3642.2003.00049.x

Wilke T. \& Davis G.M. 2000. Infraspecific mitochondrial sequence diversity in Hydrobia ulvae and Hydrobia ventrosa (Hydrobiidae: Rissooidea: Gastropoda): Do their different life histories affect biogeographic patterns and gene flow? Biological Journal of the Linnean Society 70: 89-105. http:// dx.doi.org/10.1111/j.1095-8312.2000.tb00202.x

Wilke T., Albrecht C., Anistratenko V.V., Sahin S.K. \& Yıldırım M.Z. 2007. Testing biogeographical hypotheses in space and time: faunal relationships of the putative ancient Lake Eğirdir in Asia Minor. Journal of Biogeography 34: 1807-1821. http://dx.doi.org/10.1111/j.1365-2699.2007.01727.x

Wilke T., Davis G.M., Falniowski A., Giusti F., Bodon M. \& Szarowska M. 2001. Molecular systematics of Hydrobiidae (Mollusca: Gastropoda: Rissooidea): testing monophyly and phylogenetic relationships. Proceedings of the Academy of Natural Sciences of Philadelphia 151:1-21. http://dx.doi. org/10.1635/0097-3157(2001)151[0001:MSOHMG]2.0.CO;2

Wilke T., Davis G.M., Qiu D.C. \& Spear R.C. 2006. Extreme mitochondrial sequence diversity in the intermediate schistosomiasis host Oncomelania hupensis robertsoni: another case of ancestral polymorphism? Malacologia 48: 143-157. Available from http://biodiversitylibrary.org/page/13115586 [accessed 30 May 2016]

Wilke T., Haase M., Hershler R., Liu H.P., Misof B. \& Ponder W. 2013. Pushing short DNA fragments to the limit: Phylogenetic relationships of 'hydrobioid' gastropods (Caenogastropoda: Rissooidea). Molecular Phylogenetics and Evolution 66: 715-736. http://dx.doi.org/10.1016/j.ympev.2012.10.025

Wilke T., Pfenninger M. \& Davis G.M. 2002. Anatomical variation in cryptic mudsnail species: Statistical discrimination and evolutionary significance. Proceedings of the National Academy of Sciences of Philadelphia 152: 45-66.

Wilke T., Rolán E. \& Davis G.M. 2000. The mudsnail genus Hydrobia s.s. in the northern Atlantic and western Mediterranean: a phylogenetic hypothesis. Marine Biology 137: 827-833. http://dx.doi. org/10.1007/s002270000407

Yıldırım M.Z., Koca S.B. \& Kebapçi Ü. 2006. Supplement to the Prosobranchia (Mollusca: Gastropoda) fauna of fresh and brackish waters of Turkey. Turkish Journal of Zoology 30: 197-204.

Manuscript received: 25 October 2015

Manuscript accepted: 9 March 2016

Published on: 28 June 2016

Topic editor: Rudy Jocqué

Desk editor: Kristiaan Hoedemakers

Printed versions of all papers are also deposited in the libraries of the institutes that are members of the EJT consortium: Muséum national d'Histoire naturelle, Paris, France; Botanic Garden Meise, Belgium; Royal Museum for Central Africa, Tervuren, Belgium; Natural History Museum, London, United Kingdom; Royal Belgian Institute of Natural Sciences, Brussels, Belgium; Natural History Museum of Denmark, Copenhagen, Denmark; Naturalis Biodiversity Center, Leiden, the Netherlands. 